\title{
Worldwide variations in the prevalence of asthma symptoms: the International Study of Asthma and Allergies in Childhood (ISAAC)
}

\author{
The International Study of Asthma and Allergies in Childhood (ISAAC) Steering Committee*
}

Worldwide variations in the prevalence of asthma symptoms: the International Study of Asthma and Allergies in Childhood (ISAAC). ISAAC Steering Committee.

ABSTRACT: The International Study of Asthma and Allergies in Childhood (ISAAC) was designed to allow comparisons between populations in different countries.

ISAAC Phase One, reported here, used standardized simple surveys which were conducted among representative samples of school children from centres in most regions of the world.

Two age groups (13-14 and 6-7 yrs) with approximately 3,000 children in each group were studied in each centre. The 13-14 yr olds $(n=463,801)$ were studied in 155 centres (56 countries) and the 6-7 yr olds $(n=257,800)$ were studied in 91 centres $(38$ countries).

There were marked variations in the prevalence of asthma symptoms with up to 15-fold differences between countries. The prevalence of wheeze in the last 12 months ranged from $2.1-32.2 \%$ in the older age group and 4.1-32.1\% in the younger age group and was particularly high in English speaking countries and Latin America. A video questionnaire completed in the older age group in 99 centres (42 countries) showed a similar pattern.

The major differences between populations found in the International Study of Asthma and Allergies in Childhood Phase One are likely to be due to environmental factors. The results provide a framework for studies between populations in contrasting environments which are likely to yield new clues about the aetiology of asthma. Eur Respir J 1998; 12: 315-335.

\author{
*Members of the Writing Group and \\ Steering Committee listed at end of \\ paper \\ Correspondence: M.I. Asher \\ Dept of Paediatrics \\ Faculty of Medicine and Health Sciences \\ University of Auckland \\ Private Bag 92019 \\ Auckland \\ New Zealand \\ Fax: 6493737486 \\ Keywords: Allergies \\ asthma \\ childhood \\ ISAAC \\ video \\ wheezing
}

Received: September 11997

Accepted after revision March 251998
There is considerable concern that the prevalence of asthma and allergic diseases is increasing in Western and developing countries. However, the aetiology of these conditions remains poorly understood, despite a large volume of clinical and epidemiological research within populations that has been directed at explaining why some individuals and not others develop asthma and allergies. Investigation of the reasons for variations in prevalence between populations may be a more fertile source of new aetiological clues, but little is known about worldwide variations in the prevalence of asthma and allergic diseases. Phase One of the International Study of Asthma and Allergies in Childhood (ISAAC) developed simple methods for measuring the prevalence of childhood asthma, allergic rhinitis and atopic eczema for international comparisons, suitable for different geographical locations and languages $[1,2]$. ISAAC is a unique project which has attracted worldwide interest and large scale participation.

The aims of ISAAC Phase One were: 1) to describe the prevalence and severity of asthma, rhinitis and eczema in children living in different centres and to make comparisons within and between countries; 2) to obtain baseline measures for assessment of future trends in the prevalence and severity of these diseases; and 3) to provide a framework for further aetiological research into lifestyle, envi- ronmental, genetic and medical care factors affecting these diseases.

This publication describes the results of the worldwide ISAAC study of the prevalence of symptoms of asthma. The prevalence of symptoms of allergic rhinoconjunctivitis and atopic eczema, and an overview of all three conditions are described in other publications $[3,4]$.

\section{Materials and methods}

ISAAC Phase One uses core questionnaires designed to assess the prevalence and severity of asthma and allergic disease in defined populations. The methods are described in the ISAAC manual and a previous paper concerning rationale and methods [2]. Each centre obtained the necessary approval from an ethics committee before the start of the study.

\section{Collaborating centres and registration}

ISAAC was open to any collaborator who agreed to adhere to the protocol. Countries in which there was little existing information about asthma and allergies were particularly encouraged to participate. Each collaborating centre was responsible for completing a registration document 
and obtaining its own funding. Included in this first worldwide analysis are a total of 155 centres for the 13-14 yr old age group and 91 centres for the 6-7 yr old age group. For each centre included, the data were received and verified by the ISAAC International Data Centre by June 30, 1996. The data-checking processes and a detailed report on study design and methods were completed by November 24, 1997. The first centre began its survey in 1991, but most centres undertook the data collection in 1994 and 1995. A small number of other centres, where the ISAAC questionnaire was used, are not included in this worldwide analysis. Reasons included that the data checking and report were not completed or because of low number of participating children $(<1,000)$, low response rate of children within schools, or other important departures from the protocol.

\section{Sampling frame}

The sampling frame was schoolchildren of two age groups (13-14 and 6-7 yrs old) within a specified geographical area (ISAAC centre), for which a detailed map was provided. The ISAAC centre was most commonly an urban area (a city) and therefore may not be representative of the country. Within centres there was no attempt to select affluent versus poor districts. A minority of ISAAC centres were regions within a country (e.g. in the UK), or a whole country (e.g. Costa Rica, Malta and Republic of Ireland). In a small number of the 246 centre/age groups there was further definition of the sampling frame by school type (17\%), ethnic group (1\%) and language (3\%). In $31 \%$ of centres some schools were excluded from the sampling frame before selection or excluded after selection for a variety of reasons, including recent participation in a similar study, children with disabilities unable to complete the questionnaires and very small numbers of children. The school selection within the sampling frame was representative of the geographical area, without selection by urban or rural residence or variations in socioeconomic status, and it included either all schools with children within the age group (26\% of centres) or a random sample of such schools (74\% of centres). A minority (19\%) used stratification of the sample before random selection. Among the 90 centres studying both age groups the strategies used for the sampling frame and selection of sample were similar for both age groups.

\section{Selection of subjects}

Subjects aged 13-14 yrs were selected because they were able to self-complete the written and video questionnaires. The younger age group, 6-7 yrs, was chosen as this is the youngest age when children are usually at school. The majority of centres $(74 \%)$ chose the school grade with children mostly of the desired age group, choosing one $(26 \%)$, two $(71 \%)$ or three or more grades $(3 \%)$. A minority of centres $(24 \%)$ chose children by their actual age, regardless of the grade within the school. The age of children was checked against the age calculated from the date of birth, but where there were inconsistencies or missing data the given age was used.

The participation rates of schools were calculated as the number of participating schools divided by the number of schools approached for participation. The response rate of children was calculated as the number of children for whom there was a completed written questionnaire in the participating schools divided by the number of children approached. A high response rate was sought and the majority of centres achieved $>80 \%$.

\section{Sample size}

The recommended sample size was 3,000 to ensure good prevalence estimates for severe asthma. For a sample size of 3,000 the $95 \%$ confidence interval (CI) around an estimate of $1 \%$ would be $0.64-1.33$ and around an estimate of 30\% would be 28.4-31.6 [5]. Centres with limited resources or small populations were included in the prevalence comparisons, providing that the sample size was at least 1,000 per age group.

\section{Phase One core questionnaire modules}

The ISAAC core questionnaires have been published previously [2], and used in a pilot study [1]. Most of the questions used for asthma symptoms are based on questions used in previous respiratory epidemiological studies and include both sensitive and specific indicators of asthma [6]. Questions about the date of completion of the questionnaire, child's date of birth, age and sex were also included. Some centres added questions on ethnicity and other questions (e.g. on environment) after the core questions, but analyses of these are not included in this paper.

Written questionnaire. Use of written questionnaires on wheezing, allergic rhinitis and atopic eczema for the 13$14 \mathrm{yr}$ old group was a requirement for participation in ISAAC Phase One. Written questionnaires to parents for the 6-7 yr old group were strongly recommended and used in $59 \%$ of centres. The questionnaires were completed by the children in the older age group and by the parents in the younger age group. The severity of asthma symptoms was assessed by three questions that asked about the following symptoms in the last 12 months: number of attacks of wheezing; sleep disturbed due to wheezing; and wheezing severe enough to limit speech to only one or two words at a time between breaths.

Translation. In $81 \%$ of centres the written questionnaire was translated from English into one $(88 \%)$ or more $(12 \%)$ local languages. The method of translation was standardized according to guidelines developed in Germany [7] and adapted for ISAAC (ISAAC Document 41). These guidelines included the use of translators familiar with asthma terminology ( $98 \%$ of centres that used translations), consultation with the local community $(60 \%)$ and back-translation into English by an independent translator $(99 \%)$. The written questionnaires were used in 41 different languages. English was used most commonly (32\% of centres), followed by Spanish (15\%), Italian (9\%), Hindi $(7 \%)$, Chinese $(6 \%)$, Portuguese $(6 \%)$, Marathi $(5 \%)$ and Malay (4\%) and other languages made up the remainder.

Video questionnaire. A video using the audiovisual presentation of young people with clinical symptoms and signs of asthma was completed, where possible, by the 13-14 yr old group $[8,9]$. After completing the written questionnaire, the children themselves were shown the video and 
answered questions at the time. The video elicited information without using written language terms to describe asthma symptoms. It was used in 99 centres with the original "European" English-language version in 33 centres and the revised "International" version in 66 centres. Both versions show five sequences of young persons with different manifestations of asthma. Two scenes were the same in both versions: the first scene, of a young person wheezing while at rest, and the fourth scene, of a young person waking at night with coughing. The European version showed only European children and the questions were written in English, with local verbal translation of instructions where appropriate. The International version showed scenes with children of different ethnic groups, there was no written language and the questions were asked verbally in the local language. This version has been validated in children in a Hong Kong population [10].

\section{Data management and analysis}

Information on the questionnaires was entered, by each collaborating centre or a national centre, on to the computer exactly as recorded by the child or parent (except for six centres where data was recoded to eliminate inconsistent responses). The entry of data was checked by using double entry ( $68 \%$ centres) or other acceptable data-checking methods $(24 \%)$, but $8 \%$ of centres used only single data entry. Data were transferred to the ISAAC International Data Centre (Auckland) using the protocol described in the ISAAC Coding and Data Transfer Manual.

The two age groups were analysed separately. Symptom prevalences in each centre were calculated by dividing the number of positive responses to each question by the number of completed questionnaires for the written and video questionnaires separately. Thus, apparent inconsistencies between responses to the stem and branch questions were accepted and not recoded. Basic descriptive summaries of the data were compiled by centre and country, in both age groups, along with correlations between variables. In centres where a random sample of schools was taken, the effect of cluster sampling by schools was examined in the correlations [11]. The effects of cluster sampling were small and hence ignored, allowing the use of the more robust Spearman correlations.

The within-country and between-country variances were estimated using a generalized linear mixed model in which country, and centre within country, are random effects. With this model, the ratio of the $95 \%$ CI of prevalences (between country to within country) were calculated. The ratio of the proportions responding "yes" for males and females in each centre was assessed, using the same generalized linear mixed model with the addition of sex as a fixed effect and a sex by country term to investigate any interaction [12].

\section{Quality-control measures}

The Steering Committee required documentation of the procedures for the study from each centre as a prerequisite for inclusion in publications of ISAAC worldwide results. Centres completed a Registration Document before starting the study and followed the protocol described in the published ISAAC Manual and ISAAC Coding and Data
Transfer Manual. All data submitted to the ISAAC International Data Centre were checked. Coding errors, omissions and inconsistencies in the demographic information were corrected with the assistance of the collaborator. A final report on all aspects of methodology actually used was completed for each centre and agreed between the ISAAC International Data Centre and the collaborators. This report included information about the sampling frame, sampling methods, participation rate of schools and of children within schools, the data entry method and details of translation. Some centres departed from the protocol (see footnotes to tables) but were included in the analysis if the methodology was otherwise sound.

\section{Results}

\section{Participants}

The requirements for analysis were met by 156 collaborating centres in 56 countries, with a total of 721,601 participating children. The principal investigators of the ISAAC collaborating centres are listed in the appendix. In the 13-14 yr age group, 155 centres from 56 countries participated, with a total of 463,801 participating children. The video questionnaire was completed in 99 collaborating centres in 42 countries, with a total of 304,796 children. For the 6-7 yr olds there were 91 collaborating centres in 38 countries, with a total of 257,800 participating children.

Information on the study sample is published elsewhere $[3,4]$. More than $85 \%$ of centres had a high rate of both participation by schools and response rate by pupils; in $<10 \%$ of centres there was a high rate of nonparticipation by schools, but there was a good response by pupils within schools. Participation rates of schools in the older age group averaged 94\% (range 37-100\%), and in the younger age group averaged $97 \%$ (range $57-100 \%$ ). Response rates of children within participating schools in the older age group averaged $92 \%$ (range 67-100\%) and in the younger age group averaged $89 \%$ (range 61-100\%).

The age range of children within each age group varied because of variations in methods of selection within schools. In the older age group $85 \%$ of centres had more than $70 \%$ of children aged 13 and 14 yrs. In the younger age group $93 \%$ of centres had more than $70 \%$ of children aged 6 and 7 yrs. In neither age group did the variations in age affect the general pattern of results.

\section{Written questionnaires}

13-14 yr old group. The prevalence rates for symptoms of asthma are presented by centre and country in table 1 . Thirty countries were represented by more than one centre. The geographic distribution of prevalence is illustrated, by centre on a world map (fig. 1), for positive answers to the question "Have you had wheezing or whistling in the chest in the last 12 months?". For this question there was a very wide range of prevalences, up to 15 -fold differences, between countries, ranging from $2.1-4.4 \%$ in Albania, China, Greece, Georgia, Indonesia, Romania and Russia, to $29.1-32.2 \%$ in Australia, New Zealand, Republic of Ireland and the UK. These countries also tended to be at the extremes for the other questions. The two centres at the extremes, Akola (1.6\%) and Scotland (36.7\%), had 
Table 1. - Summary of self-completed wheezing questionnaire (written) data, 13-14 yr old age group

\begin{tabular}{|c|c|c|c|c|c|c|c|c|}
\hline \multirow[t]{2}{*}{ Centre $^{+}$} & \multicolumn{6}{|c|}{ 12-month prevalence } & \multirow[b]{2}{*}{$\begin{array}{l}\text { Ever had } \\
\text { asthma }\end{array}$} & \multirow[b]{2}{*}{$\mathrm{n}$} \\
\hline & Wheeze & Š4 Attacks & $\begin{array}{c}\text { Wheeze } \\
\text { disturbs } \\
\text { sleep }\end{array}$ & $\begin{array}{l}\text { Severe wheeze } \\
\text { limiting speech }\end{array}$ & $\begin{array}{l}\text { Exercise } \\
\text { wheeze }\end{array}$ & $\begin{array}{l}\text { Night } \\
\text { cough }\end{array}$ & & \\
\hline \multicolumn{9}{|l|}{ Africa } \\
\hline Ethiopia & 6.2 & 3.2 & 1.4 & 4.1 & 13.9 & 14.5 & 2.5 & 5978 \\
\hline Addis Ababa & 10.7 & 5.6 & 2.5 & 7.3 & 27.2 & 27.3 & 2.8 & 2951 \\
\hline $\mathrm{Jima}^{\mathrm{c}, \mathrm{f}}$ & 1.9 & 0.9 & 0.3 & 1.0 & 1.0 & 2.0 & 2.2 & 3027 \\
\hline Kenya & 13.9 & 3.8 & 4.2 & 5.8 & 24.0 & 27.3 & 11.2 & 6267 \\
\hline Eldoret & 10.4 & 2.3 & 1.9 & 3.0 & 17.4 & 18.4 & 6.8 & 3024 \\
\hline Nairobi & 17.1 & 5.2 & 6.3 & 8.4 & 30.2 & 35.6 & 15.4 & 3243 \\
\hline \multicolumn{9}{|l|}{ Nigeria } \\
\hline Ibadan & 10.7 & 3.2 & 3.7 & 7.6 & 43.4 & 31.7 & 18.4 & 3057 \\
\hline South Africa & & & & & & & & \\
\hline Cape Town & 16.1 & 3.3 & 3.6 & 5.2 & 21.4 & 23.6 & 13.1 & 5173 \\
\hline $\begin{array}{l}\text { Regional total } \\
\text { Africa (French speaking) }\end{array}$ & 11.7 & 3.4 & 3.1 & 5.4 & 23.3 & 23.3 & 10.2 & 20475 \\
\hline \multicolumn{9}{|l|}{ Algeria } \\
\hline Algiers ${ }^{f}$ & 7.8 & 2.0 & 1.8 & 3.5 & 13.6 & 19.6 & 6.4 & 1173 \\
\hline $\begin{array}{l}\text { Regional total } \\
\text { Asia-Pacific }\end{array}$ & 7.8 & 2.0 & 1.8 & 3.5 & 13.6 & 19.6 & 6.4 & 1173 \\
\hline China & 4.2 & 0.9 & 0.3 & 0.7 & 21.7 & 15.0 & 6.1 & 19008 \\
\hline Beijing & 5.1 & 1.5 & 0.2 & 0.9 & 25.2 & 15.7 & 6.9 & 4167 \\
\hline Chongqing & 4.7 & 0.8 & 0.2 & 0.4 & 25.1 & 11.1 & 7.1 & 4296 \\
\hline Guangzhou & 3.4 & 0.7 & 0.3 & 0.5 & 17.4 & 18.9 & 3.9 & 3855 \\
\hline Shanghai & 3.3 & 0.4 & 0.2 & 0.7 & 15.8 & 6.9 & 7.1 & 3483 \\
\hline Wulumuqi & 4.0 & 1.0 & 0.3 & 0.8 & 24.3 & 23.4 & 5.4 & 3207 \\
\hline Hong Kong & & & & & & & & \\
\hline $\begin{array}{l}\text { Hong Kong } \\
\text { Indonesia }\end{array}$ & 12.4 & 4.4 & 0.5 & 2.4 & 29.1 & 27.7 & 11.2 & 4666 \\
\hline $\begin{array}{l}\text { Indonesia } \\
\text { Bandung }\end{array}$ & 2.1 & 0.4 & 0.8 & 0.9 & 2.3 & 4.0 & 1.6 & 2249 \\
\hline Japan & & & & & & 4.0 & 1.0 & \\
\hline Fukuoka & 13.4 & 4.2 & 0.6 & 2.1 & 27.3 & 14.0 & 18.9 & 2831 \\
\hline Malaysia & 9.6 & 2.7 & 0.8 & 1.8 & 14.0 & 24.0 & 10.9 & 18636 \\
\hline Alor Setar ${ }^{\mathrm{d}}$ & 8.9 & 2.1 & 0.4 & $\begin{array}{l}1.0 \\
1.8\end{array}$ & 16.4 & 28.3 & 10.2 & 3298 \\
\hline $\mathrm{Ipoh}^{\mathrm{f}, \mathrm{h}}$ & 10.0 & 2.8 & 0.8 & 1.7 & 10.3 & 23.4 & 11.4 & 3313 \\
\hline Klang Valley & 12.3 & 3.8 & 1.0 & 2.3 & 15.7 & 25.3 & 13.9 & 6079 \\
\hline Kota Bharu & 6.8 & 1.7 & 0.4 & 1.3 & 9.9 & 21.6 & 8.6 & 3113 \\
\hline Muar $^{\mathrm{f}}$ & 7.1 & 2.0 & 1.0 & 1.4 & 16.4 & 19.4 & 7.4 & 2833 \\
\hline Philippines & & & & & & & & \\
\hline Metro Manilla & 12.3 & 2.4 & 2.0 & 4.1 & 21.4 & 34.3 & 17.6 & 3207 \\
\hline $\begin{array}{l}\text { Singapore } \\
\text { Singapore }\end{array}$ & & & & & & & & \\
\hline $\begin{array}{l}\text { Singapore } \\
\text { South Korea }\end{array}$ & & 2.1 & 1.3 & & 13.2 & 11.3 & 20.9 & 4206 \\
\hline South Korea & 7.7 & 1.6 & 0.2 & 2.7 & 12.4 & $\begin{array}{l}8.6 \\
83\end{array}$ & 2.4 & 9983 \\
\hline $\begin{array}{l}\text { Provincial Korea }{ }^{\mathrm{a}, \mathrm{d}} \\
\text { Seoulla, }\end{array}$ & $\begin{array}{l}7.5 \\
8.3\end{array}$ & $\begin{array}{l}1.5 \\
2.0\end{array}$ & $\begin{array}{l}0.2 \\
0.3\end{array}$ & 2.7 & 11.9 & $\begin{array}{l}8.3 \\
9.2\end{array}$ & $\begin{array}{l}2.2 \\
28\end{array}$ & 6990 \\
\hline $\begin{array}{l}\text { Seoula, d } \\
\text { Taiwan }\end{array}$ & & & 0.3 & 2.6 & & 9.2 & & 2993 \\
\hline Taipei ${ }^{\mathrm{a}}$ & 5.2 & 1.6 & 0.4 & 0.8 & 8.2 & 10.4 & 9.0 & 11400 \\
\hline Thailand & 13.0 & 4.0 & 1.1 & 3.5 & 14.5 & 28.4 & 11.6 & 7640 \\
\hline Bangkok ${ }^{b}$ & 13.5 & 4.1 & 1.3 & 3.9 & 15.6 & 28.4 & 14.1 & 3713 \\
\hline Chiang Mai & 12.6 & 3.8 & 1.0 & 3.2 & 13.5 & 28.5 & 9.3 & 3927 \\
\hline Regional total & 8.0 & 2.2 & 0.6 & 1.8 & 16.0 & 17.8 & 9.4 & 83826 \\
\hline Eastern Mediterranean & & & & & & & & \\
\hline Iran & 10.9 & 2.3 & 1.8 & 2.6 & 11.3 & 13.8 & 2.7 & 5873 \\
\hline Rasht & 9.7 & 2.3 & 1.7 & 2.3 & 9.1 & 13.2 & 2.7 & 3182 \\
\hline Tehran & 12.4 & 2.2 & 2.0 & 3.0 & 13.8 & 14.6 & 2.6 & 2691 \\
\hline Kuwait & & & & & & & & \\
\hline Kuwait ${ }^{b, f}$ & 17.0 & 6.3 & 5.7 & 10.6 & 24.7 & 30.5 & 17.5 & 1056 \\
\hline $\begin{array}{l}\text { Lebanon } \\
\text { Beirut }\end{array}$ & 144 & 41 & 50 & 70 & 161 & 27 & 116 & \\
\hline $\begin{array}{l}\text { Beirut }{ }^{2} \\
\text { Malta }\end{array}$ & 14.4 & 4.1 & 5.0 & 7.0 & 16.1 & 22.4 & 11.6 & 2993 \\
\hline Malta & 16.0 & 4.6 & 2.4 & 3.6 & 20.6 & 31.8 & 11.1 & 4184 \\
\hline Morocco & 7.5 & 2.1 & 2.2 & 2.7 & 17.9 & 17.6 & 11.7 & 9359 \\
\hline Casablanca & 9.9 & 3.1 & 3.0 & 3.4 & 18.9 & 19.8 & 12.1 & 3183 \\
\hline Marrakech & 5.6 & 1.5 & 1.0 & 2.2 & 21.1 & 18.6 & 17.1 & 2900 \\
\hline Rabat & 6.8 & 1.7 & 2.7 & 2.4 & 14.2 & 14.5 & 6.6 & 3276 \\
\hline Oman & & & & & & & & \\
\hline Al-Khod & 8.9 & 2.6 & 2.9 & 4.0 & 19.2 & 20.9 & 20.7 & 3174 \\
\hline Pakistan & & & & & & & & \\
\hline Karachi & 8.5 & 2.0 & 1.6 & 3.9 & 15.1 & 17.6 & 7.3 & 1829 \\
\hline $\begin{array}{l}\text { Regional total } \\
\text { Latin America }\end{array}$ & 10.7 & 2.9 & 2.6 & 3.8 & 16.9 & 20.2 & 10.7 & 28468 \\
\hline Argentina & 10.9 & 3.1 & 2.1 & 3.8 & 15.1 & 39.1 & 7.3 & 6004 \\
\hline Buenos Aires & 9.9 & 2.8 & 1.9 & 3.1 & 13.8 & 36.2 & 6.6 & 2996 \\
\hline Rosario & 11.8 & 3.4 & 2.4 & 4.4 & 16.5 & 41.9 & 7.9 & 3008 \\
\hline Brazil & 22.7 & 4.4 & 3.7 & 4.7 & 23.6 & 32.6 & 14.9 & 15454 \\
\hline Curitiba & 18.4 & 3.5 & 2.7 & 4.6 & 19.8 & 30.1 & 8.6 & 3004 \\
\hline Porto Alegre & 24.7 & 4.5 & 4.5 & 5.7 & 29.0 & 39.2 & 21.9 & 3195 \\
\hline Recife & 19.7 & 3.8 & 4.6 & 4.8 & 20.5 & 30.9 & 20.9 & 3086 \\
\hline Salvador $^{\mathrm{f}}$ & 27.0 & 6.0 & 3.0 & 5.4 & 27.6 & 29.6 & 12.5 & 3162 \\
\hline São Paulof & 23.3 & 4.4 & 3.5 & 2.7 & 20.5 & 33.0 & 10.0 & 3007 \\
\hline Chile & 10.2 & 1.5 & 1.4 & 2.5 & 15.9 & 21.6 & 10.7 & 12708 \\
\hline Central Santiago ${ }^{\mathrm{i}}$ & 11.7 & 1.7 & 1.3 & 2.0 & 15.0 & 23.1 & 12.4 & 2944 \\
\hline Punta Arenas & 6.8 & 1.1 & 0.8 & 1.3 & 6.3 & 15.5 & 7.3 & 3482 \\
\hline
\end{tabular}


Table 1. - continued.

\begin{tabular}{|c|c|c|c|c|c|c|c|c|}
\hline \multirow[t]{2}{*}{ Centre $^{+}$} & \multicolumn{6}{|c|}{ 12-month prevalence } & \multirow[b]{2}{*}{$\begin{array}{l}\text { Ever had } \\
\text { asthma }\end{array}$} & \multirow[b]{2}{*}{$\mathrm{n}$} \\
\hline & Wheeze & Š4 Attacks & $\begin{array}{l}\text { Wheeze } \\
\text { disturbs } \\
\text { sleep }\end{array}$ & $\begin{array}{l}\text { Severe wheeze } \\
\text { limiting speech }\end{array}$ & $\begin{array}{l}\text { Exercise } \\
\text { wheeze }\end{array}$ & $\begin{array}{l}\text { Night } \\
\text { cough }\end{array}$ & & \\
\hline South Santiago & 11.1 & 1.2 & 2.2 & 4.9 & 25.3 & 32.8 & 11.5 & 3051 \\
\hline Valdivia & 11.5 & 2.1 & 1.2 & 2.2 & 18.0 & 16.2 & 11.9 & 3231 \\
\hline \multicolumn{9}{|l|}{ Costa Rica } \\
\hline $\begin{array}{l}\text { Costa Rica } \\
\text { Mexico }\end{array}$ & 23.7 & 5.3 & 3.5 & 10.1 & 24.2 & 31.1 & 18.5 & 3200 \\
\hline Cuernavacaj & 6.6 & 1.5 & 1.6 & 2.4 & 4.0 & 13.6 & 5.5 & 3102 \\
\hline $\begin{array}{l}\text { Panama } \\
\text { David-Panamáf }\end{array}$ & 176 & 3.4 & 2.0 & 67 & 14.6 & 217 & 169 & 2885 \\
\hline Paraguay & & & & & & & & \\
\hline Asunción & 19.4 & 3.4 & 3.0 & 5.2 & 15.3 & 31.3 & 12.2 & 2966 \\
\hline $\begin{array}{l}\text { Peru } \\
\text { Lima }\end{array}$ & 26.0 & 4.8 & 3.3 & 69 & 350 & 336 & 280 & 3158 \\
\hline Uruguay & 20.0 & 4.0 & $3 . J$ & 0.7 & 30.9 & 35.0 & 20.0 & סוJנ \\
\hline Montevideo ${ }^{f}$ & 19.0 & 5.4 & 2.6 & 5.1 & 18.4 & 28.1 & 15.3 & 3072 \\
\hline Regional total & 16.9 & 3.4 & 2.6 & 4.5 & 19.1 & 28.6 & 13.4 & 52549 \\
\hline North America & & & & & & & & \\
\hline Canada & 28.1 & 9.1 & 2.1 & 8.1 & 34.3 & 36.3 & 16.5 & 4952 \\
\hline Hamilton ${ }^{b}$ & 30.6 & 10.0 & 2.2 & 8.9 & 36.5 & 37.5 & 19.2 & 3051 \\
\hline Saskatoon ${ }^{b}$ & 24.0 & 7.7 & 2.0 & 6.7 & 30.8 & 34.4 & 12.2 & 1901 \\
\hline USA & 21.7 & 6.5 & 4.2 & 10.0 & 28.7 & 31.9 & 16.5 & 7508 \\
\hline Chicago (3) $)^{\mathrm{a}, \mathrm{g}, \mathrm{h}}$ & 24.6 & 8.8 & 3.0 & 5.5 & 27.4 & 29.4 & 17.8 & 1422 \\
\hline Chicago (4)g & 19.8 & 5.6 & 5.9 & 13.3 & 29.5 & 35.5 & 16.1 & 3756 \\
\hline Seattle $\mathrm{a}$ & 22.9 & 6.7 & 2.4 & 7.4 & 28.2 & 27.6 & 16.4 & 2330 \\
\hline Regional total & 24.2 & 7.6 & 3.4 & 9.2 & 30.9 & 33.7 & 16.5 & 12460 \\
\hline Northern and Eastern Europe & & & & & & & & \\
\hline Albania & & & & & & & & \\
\hline Tiranë & 2.6 & 0.3 & 0.3 & 0.8 & 4.5 & 5.7 & 1.6 & 2957 \\
\hline Estonia & 10.8 & 1.7 & 1.1 & 2.1 & 12.6 & 15.6 & 3.0 & 4984 \\
\hline Narva & 16.7 & 2.0 & 2.2 & 4.0 & 21.2 & 18.6 & 2.5 & 1424 \\
\hline Tallinn & 8.5 & 1.5 & 0.7 & 1.3 & 9.1 & 14.4 & 3.3 & 3560 \\
\hline Finland & 16.0 & 3.5 & 0.5 & 3.1 & 20.6 & 16.5 & 6.6 & 11895 \\
\hline Helsinki & 19.8 & 5.1 & 0.8 & 5.1 & 25.1 & 19.5 & 7.4 & 2855 \\
\hline Kuopio County & 13.1 & 2.8 & 0.6 & 2.0 & 17.6 & 14.5 & 4.6 & 2878 \\
\hline Lapland Area & 16.1 & 3.3 & 0.2 & 2.7 & 20.6 & 15.6 & 6.6 & 3077 \\
\hline Turku and Pori County & 15.0 & 2.9 & 0.5 & 2.7 & 19.2 & 16.3 & 7.8 & 3085 \\
\hline Georgia & 3.6 & 0.7 & 0.7 & 0.8 & 6.4 & 7.8 & 3.1 & 6746 \\
\hline Kutaisi & 3.6 & 0.7 & 0.5 & 0.7 & 8.2 & 9.0 & 3.8 & 3297 \\
\hline Tbilisi & 3.7 & 0.8 & 0.8 & 1.0 & 4.7 & 6.6 & 2.4 & 3449 \\
\hline Latvia & 8.4 & 1.2 & 0.9 & 1.6 & 10.7 & 14.8 & 4.3 & 6149 \\
\hline Riga & 8.3 & 1.1 & 0.7 & 1.4 & 8.6 & 12.3 & 3.9 & 3004 \\
\hline Rural Latvia & 8.6 & 1.4 & 1.2 & 1.8 & 12.8 & 17.3 & 4.6 & 3145 \\
\hline Poland & 8.1 & 1.9 & 0.9 & 1.7 & 9.3 & 14.8 & 2.4 & 10167 \\
\hline Krakow (1993) & 8.8 & 2.1 & 0.7 & 1.9 & 9.7 & 15.9 & 2.9 & 3750 \\
\hline Krakow (1995) & 7.5 & 1.9 & 0.9 & 1.8 & 9.9 & 16.1 & 2.3 & 2786 \\
\hline Poznan & 7.9 & 1.7 & 1.0 & 1.5 & 8.3 & 12.6 & 2.0 & 3631 \\
\hline Romania & & & & & & & & \\
\hline Cluj & 3.0 & 0.7 & 0.4 & 0.7 & 6.9 & 4.4 & 3.7 & 3396 \\
\hline Russia & & & & & & & & \\
\hline Moscow & 4.4 & 0.7 & 0.1 & 0.8 & 6.6 & 5.6 & 2.4 & 3411 \\
\hline Sweden & 12.9 & 3.9 & 0.8 & 2.0 & 19.3 & 12.0 & 10.4 & 6452 \\
\hline Linköping & 11.2 & 3.5 & 0.7 & 1.7 & 17.4 & 10.2 & 10.0 & 3377 \\
\hline Stockholm/Uppsala & 14.8 & 4.5 & 0.9 & 2.3 & 21.4 & 14.0 & 10.8 & 3075 \\
\hline Uzbekistan & 9.2 & 0.7 & 0.2 & 2.1 & 11.1 & 9.7 & 1.7 & 4662 \\
\hline Samarkand $\mathrm{d}^{\mathrm{f}}$ & 14.4 & 1.1 & 0.3 & 3.8 & 15.1 & 15.8 & 2.3 & 1758 \\
\hline Tashkent ${ }^{\mathrm{f}}$ & 6.0 & 0.5 & 0.1 & 1.1 & 8.7 & 6.1 & 1.4 & 2904 \\
\hline Regional total & 9.2 & 1.9 & 0.6 & 1.8 & 12.3 & 12.2 & 4.4 & 60819 \\
\hline Oceania & & & & & & & & \\
\hline Australia & 29.4 & 10.0 & 3.0 & 8.3 & 37.0 & 28.3 & 28.2 & 12278 \\
\hline Adelaide & 33.5 & 12.8 & 3.3 & 8.7 & 41.4 & 27.8 & 30.4 & 3030 \\
\hline Melbourne & 27.3 & 9.9 & 3.3 & 8.2 & 35.7 & 27.7 & 26.6 & 2759 \\
\hline Perth ${ }^{\mathrm{h}}$ & 31.4 & 9.6 & 2.3 & 8.7 & 38.9 & 28.6 & 30.2 & 3650 \\
\hline Sydney & 24.7 & 7.5 & 3.1 & 7.3 & 31.0 & 29.0 & 24.8 & 2839 \\
\hline New Zealand & 30.2 & 9.9 & 3.2 & 8.0 & 40.3 & 30.0 & 24.4 & 19023 \\
\hline Auckland & 26.5 & 8.0 & 2.7 & 8.1 & 36.1 & 29.7 & 22.9 & 3206 \\
\hline Bay of Plenty & 29.5 & 9.0 & 3.3 & 7.1 & 39.4 & 31.3 & 22.3 & 2813 \\
\hline Christchurch & 29.6 & 9.7 & 2.9 & 7.5 & 40.3 & 27.4 & 25.9 & 3191 \\
\hline Hawke's Bay & 32.4 & 11.0 & 4.6 & 8.6 & 42.4 & 33.2 & 25.7 & 3550 \\
\hline Nelson & 30.9 & 10.2 & 2.6 & 8.2 & 43.3 & 26.3 & 20.2 & 1839 \\
\hline Wellington & 31.6 & 11.1 & 3.0 & 8.3 & 41.1 & 30.3 & 26.3 & 4424 \\
\hline Regional total & 29.9 & 9.9 & 3.1 & 8.1 & 39.0 & 29.3 & 25.9 & 31301 \\
\hline South-east Asia & & & & & & & & \\
\hline India & 6.0 & 1.6 & 1.1 & 3.0 & 9.5 & 14.1 & 4.5 & 37171 \\
\hline Akola & 1.6 & 0.5 & 0.5 & 1.0 & 2.7 & 3.8 & 2.6 & 2138 \\
\hline Bombay (16) & 1.9 & 1.2 & 0.8 & 1.0 & 2.6 & 6.5 & 3.6 & 4225 \\
\hline Bombay $(17)^{\mathrm{h}}$ & 10.6 & 1.8 & 1.3 & 3.2 & 11.1 & 22.4 & 6.5 & 2226 \\
\hline Bombay (18) & 3.6 & 1.0 & 0.8 & 1.4 & 7.4 & 14.9 & 5.2 & 3178 \\
\hline Borivali & 3.4 & 0.6 & 0.4 & 1.6 & 5.3 & 10.2 & 5.9 & 3878 \\
\hline Chandigarh & 4.2 & 1.5 & 0.7 & 2.7 & 8.0 & 8.0 & 3.3 & 3139 \\
\hline Jodhpur & 10.7 & 3.5 & 1.7 & 4.8 & 15.9 & 18.4 & 6.4 & 1094 \\
\hline Kottayam & 17.8 & 1.7 & 1.8 & 13.5 & 17.9 & 32.2 & 12.4 & 2047 \\
\hline
\end{tabular}


Table 1. - continued.

\begin{tabular}{|c|c|c|c|c|c|c|c|c|}
\hline \multirow[t]{2}{*}{ Centre $^{+}$} & \multicolumn{6}{|c|}{ 12-month prevalence } & \multirow[b]{2}{*}{$\begin{array}{l}\text { Ever had } \\
\text { asthma }\end{array}$} & \multirow[b]{2}{*}{$\mathrm{n}$} \\
\hline & Wheeze & Š4 Attacks & $\begin{array}{c}\text { Wheeze } \\
\text { disturbs } \\
\text { sleep }\end{array}$ & $\begin{array}{l}\text { Severe wheeze } \\
\text { limiting speech }\end{array}$ & $\begin{array}{l}\text { Exercise } \\
\text { wheeze }\end{array}$ & $\begin{array}{l}\text { Night } \\
\text { cough }\end{array}$ & & \\
\hline Madras (2) & 8.4 & 1.9 & 1.5 & 2.9 & 7.7 & 14.6 & 2.8 & 1903 \\
\hline Madras $(3)^{\mathrm{h}}$ & 6.0 & 3.3 & 2.0 & 3.6 & 7.4 & 11.5 & 1.8 & 3086 \\
\hline New Delhi & 13.0 & 3.0 & 1.9 & 4.8 & 18.4 & 25.8 & 5.3 & 3026 \\
\hline Neyveli & 6.0 & 1.9 & 1.0 & 2.5 & 23.2 & 16.9 & 2.4 & 3281 \\
\hline Orissa & 3.8 & 0.8 & 0.8 & 2.1 & 6.8 & 13.5 & 2.8 & 1248 \\
\hline Pune & 1.8 & 0.8 & 0.7 & 1.3 & 4.0 & 9.4 & 4.9 & 2702 \\
\hline Regional total & 6.0 & 1.6 & 1.1 & 3.0 & 9.5 & 14.1 & 4.5 & 37171 \\
\hline \multicolumn{9}{|l|}{ Western Europe } \\
\hline Austria & 11.6 & 2.5 & 0.8 & 3.6 & 18.0 & 13.3 & 6.0 & 4886 \\
\hline Salzburg & 11.5 & 2.3 & 0.8 & 3.0 & 17.5 & 13.3 & 6.3 & 3371 \\
\hline Urfahr-Ümgebung & 11.7 & 3.0 & 0.9 & 5.0 & 19.0 & 13.3 & 5.3 & 1515 \\
\hline \multicolumn{9}{|l|}{ Belgium } \\
\hline Antwerp & 12.0 & 3.5 & 1.5 & 2.6 & 13.1 & 21.2 & 8.1 & 1515 \\
\hline France & 13.5 & 4.1 & 1.1 & 2.8 & 20.8 & 26.5 & 12.6 & 18544 \\
\hline Marseilles & 14.9 & 4.0 & 1.2 & 2.8 & 22.8 & 26.9 & 14.4 & 3494 \\
\hline Montpellier & 18.2 & 6.6 & 1.9 & 4.2 & 25.4 & 29.8 & 14.2 & 3384 \\
\hline Pessaca,b & 12.8 & 3.9 & 1.0 & 1.8 & 19.3 & 24.3 & 15.0 & 3302 \\
\hline Strasbourg & 10.2 & 2.6 & 0.6 & 2.4 & 17.9 & 26.2 & 10.1 & 5403 \\
\hline West Marne & 13.3 & 4.2 & 1.4 & 2.9 & 20.1 & 25.4 & 10.7 & 2961 \\
\hline Germany & 13.8 & 3.4 & 1.2 & 5.7 & 22.0 & 20.8 & 5.7 & 7172 \\
\hline Greifswald & 13.3 & 3.0 & 1.1 & 5.1 & 23.0 & 21.5 & 4.2 & 3169 \\
\hline Münster & 14.1 & 3.7 & 1.3 & 6.1 & 21.2 & 20.2 & 7.0 & 4003 \\
\hline \multicolumn{9}{|l|}{ Greece } \\
\hline Athens & 3.7 & 0.7 & 0.7 & 0.7 & 3.6 & 6.7 & 4.5 & 2561 \\
\hline Italy & 8.9 & 2.0 & 0.6 & 2.0 & 14.5 & 21.2 & 9.9 & 26477 \\
\hline Ascoli Piceno & 2.7 & 0.7 & 0.4 & 0.8 & 5.5 & 9.8 & 7.7 & 1130 \\
\hline Cosenza & 7.1 & 1.4 & 0.0 & 2.3 & 14.8 & 22.4 & 8.1 & 1068 \\
\hline Cremona & 9.2 & 1.7 & 0.7 & 1.6 & 13.2 & 19.7 & 8.1 & 1201 \\
\hline Emilia-Romagna & 10.4 & 2.6 & 0.6 & 2.0 & 17.1 & 21.5 & 10.2 & 3961 \\
\hline Empoli & 13.5 & 2.3 & 0.8 & 2.5 & 17.6 & 28.6 & 12.0 & 1046 \\
\hline Firenze & 10.5 & 2.6 & 0.9 & 2.6 & 15.7 & 21.3 & 9.6 & 1171 \\
\hline Frosinone ${ }^{\mathrm{h}}$ & 7.4 & 1.7 & 0.6 & 2.3 & 15.7 & 19.0 & 11.3 & 1147 \\
\hline Milano & 10.6 & 2.3 & 0.7 & 2.5 & 18.0 & 27.3 & 10.7 & 3373 \\
\hline Roma & 9.8 & 2.0 & 0.4 & 2.6 & 14.4 & 23.0 & 11.3 & 3323 \\
\hline Siena & 13.0 & 3.0 & 1.2 & 3.0 & 20.8 & 26.4 & 11.0 & 1181 \\
\hline Torino & 8.6 & 2.3 & 0.6 & 2.9 & 18.8 & 23.3 & 9.4 & 1242 \\
\hline Trento $^{d}$ & 5.9 & 1.3 & 0.3 & 1.3 & 9.4 & 17.6 & 7.5 & 4426 \\
\hline Verona & 7.5 & 2.0 & 0.4 & 1.3 & 12.0 & 15.9 & 11.8 & 2208 \\
\hline Portugal & 9.5 & 2.4 & 1.4 & 2.2 & 13.9 & 17.0 & 12.1 & 10751 \\
\hline Funchal & 10.6 & 2.7 & 1.8 & 2.7 & 16.6 & 19.8 & 13.1 & 3532 \\
\hline Lisbon & 11.1 & 2.8 & 1.3 & 2.4 & 14.1 & 17.5 & 12.3 & 3030 \\
\hline Portimao ${ }^{\mathrm{h}}$ & 8.1 & 2.0 & 1.4 & 1.9 & 14.8 & 18.1 & 10.3 & 1058 \\
\hline Porto & 7.4 & 1.7 & 1.0 & 1.6 & 10.5 & 13.1 & 11.3 & 3131 \\
\hline \multicolumn{9}{|l|}{ Republic of Ireland } \\
\hline Republic of Ireland & 29.1 & 8.1 & 2.6 & 6.0 & 25.2 & 33.9 & 15.2 & 3147 \\
\hline Spain & 10.3 & 2.7 & 1.1 & 2.4 & 16.1 & 22.4 & 10.5 & 25021 \\
\hline Barcelona & 14.3 & 3.4 & 1.4 & 2.7 & 18.3 & 20.5 & 11.1 & 3031 \\
\hline Bilbao & 11.9 & 4.2 & 1.5 & 3.0 & 19.8 & 25.6 & 16.3 & 3212 \\
\hline Cartagena & 10.5 & 3.4 & 1.8 & 2.9 & 14.9 & 20.4 & 10.5 & 3017 \\
\hline Castellón & 7.1 & 1.8 & 0.5 & 1.1 & 12.8 & 22.3 & 7.8 & 3094 \\
\hline Cádiz & 15.4 & 3.2 & 1.9 & 4.2 & 20.2 & 28.0 & 11.2 & 3270 \\
\hline Pamplona & 5.5 & 1.7 & 0.5 & 1.1 & 12.2 & 17.6 & 8.7 & 3040 \\
\hline Valencia & 11.0 & 2.6 & 1.1 & 2.8 & 17.2 & 21.2 & 12.1 & 3179 \\
\hline Valladolid $^{f}$ & 6.1 & 1.5 & 0.5 & 1.4 & 13.1 & 22.9 & 6.3 & 3178 \\
\hline UK & 32.2 & 9.3 & 3.5 & 8.5 & 29.1 & 42.3 & 20.7 & 35485 \\
\hline Anglia and Oxford ${ }^{\mathrm{h}}$ & 34.3 & 10.2 & 2.9 & 9.0 & 30.3 & 43.3 & 22.8 & 2324 \\
\hline Guernsey & 35.0 & 9.6 & 4.0 & 8.6 & 31.7 & 44.9 & 21.4 & 1170 \\
\hline Isle of Man & 33.4 & 9.1 & 3.4 & 8.5 & 30.7 & 41.5 & 18.8 & 1467 \\
\hline Jersey & 35.2 & 10.7 & 2.7 & 8.5 & 30.7 & 45.1 & 18.8 & 1135 \\
\hline North East and Yorkshire & 34.1 & 9.3 & 4.3 & 8.7 & 28.7 & 47.1 & 20.3 & 3709 \\
\hline North Thames $^{\mathrm{h}}$ & 30.5 & 7.4 & 3.3 & 7.7 & 25.9 & 47.1 & 18.2 & 2220 \\
\hline North West & 30.0 & 9.0 & 3.5 & 7.9 & 26.1 & 46.1 & 19.8 & 3029 \\
\hline Scotland & 36.7 & 11.6 & 4.7 & 10.0 & 31.4 & 42.3 & 21.4 & 4444 \\
\hline South Thamesh & 31.3 & 9.7 & 3.4 & 8.8 & 26.8 & 45.5 & 20.5 & 2297 \\
\hline South and West & 35.3 & 10.9 & 3.1 & 9.6 & 30.9 & 43.1 & 20.8 & 2707 \\
\hline Sunderland & 19.9 & 6.2 & 2.4 & 5.0 & 24.2 & 21.0 & 22.2 & 2092 \\
\hline Surrey/Sussex & 26.8 & 8.6 & 2.3 & 6.9 & 38.6 & 26.6 & 19.0 & 2114 \\
\hline Trent ${ }^{\mathrm{h}}$ & 33.6 & 8.8 & 3.6 & 8.2 & 27.8 & 45.6 & 22.3 & 2207 \\
\hline Wales $^{\mathrm{h}}$ & 33.6 & 9.0 & 3.7 & 9.0 & 29.7 & 43.6 & 21.8 & 2351 \\
\hline West Midlands ${ }^{\mathrm{h}}$ & 30.0 & 8.0 & 3.7 & 8.5 & 25.1 & 46.8 & 20.7 & 2219 \\
\hline Regional total & 16.7 & 4.6 & 1.7 & 4.2 & 20.0 & 27.1 & 13.0 & 135559 \\
\hline Global total & 13.8 & 3.7 & 1.7 & 3.8 & 18.8 & 22.3 & 11.3 & 463801 \\
\hline
\end{tabular}

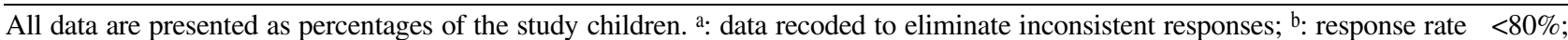
c: no age or date of birth on questionnaire; ${ }^{\mathrm{s}}$ : no age on questionnaire; ${ }^{\mathrm{e}}$ : no date of interview or date of birth on questionnaire; $\mathrm{f}$ : single data entry; $\mathrm{g}$ : no eczema questionnaire; $\mathrm{h}:<10$ schools if S 10 schools in sampling frame; ${ }^{\mathrm{i}}$ : no sex information provided; $\mathrm{j}: 90 \%$ children 11-12 yrs old. +: numbers in parenthesis indicate International Study of Asthma and Allergies in Childhood (ISAAC) registration number for that centre, as given by the national coordinator. 


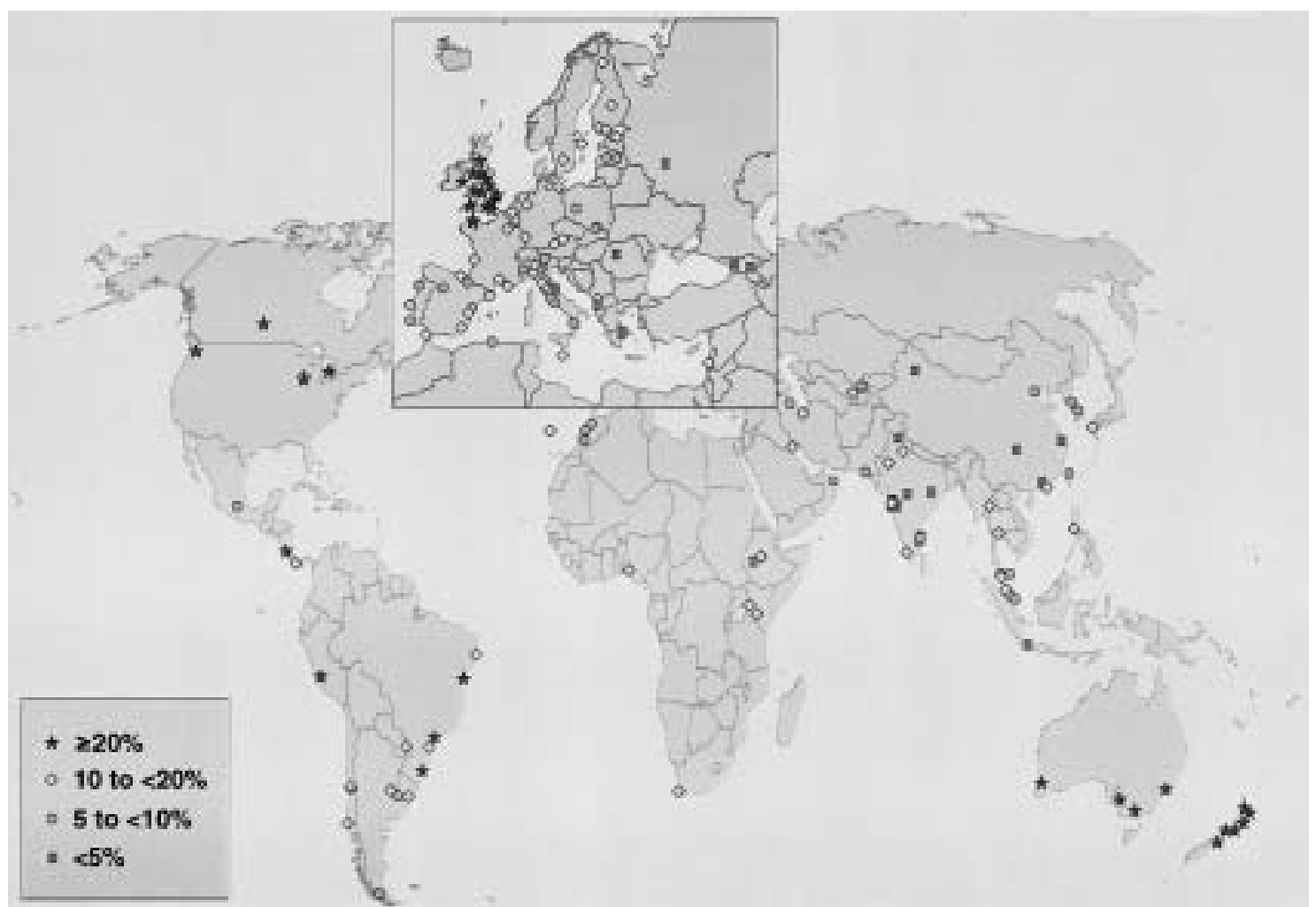

Fig. 1. - World map for the 13-14 yr old age group, showing the percentage of children who answered "yes" to the written question "Have you had wheezing or whistling in the chest in the last 12 months?". Each point represents the prevalence for one collaborating centre, with distinct colour symbols used for different prevalence ranges.

over 20 -fold differences. Other countries that had a prevalence of 12 month wheeze under 10\% were found mainly in Asia, Northern Africa, Eastern Europe and the Eastern Mediterranean regions and those over $20 \%$ were found mainly in North America, Latin America and Oceania.

The between-country variation (56 countries) was greater than the within-country variation (30 countries with more than one centre) for all questions: 12 month wheeze was 1.7 times larger; four or more attacks of wheezing in the last 12 months 2.8; waking on one or more nights a week 2.8; wheezing severe enough to limit speech to only one or two words at a time between breaths 1.9; wheeze with exercise 1.3; dry cough at night 1.5 ; and reported asthma ever 3.7 times larger.

The ranking of countries for sleep disturbance due to wheezing on S1 night week ${ }^{-1}$ in the last 12 months is illustrated in figure 2 . The seven countries with the highest prevalence of positive answers to this question were Brazil, Kenya, Kuwait, Lebanon, Nigeria, South Africa and the USA, which were not the countries ranking highest for the prevalence of 12 month wheeze. The seven countries with the lowest prevalence of positive answers to this question were Albania, China, Romania, Russia, South Korea, Taiwan and Uzbekistan, which were similar to the centres ranking lowest for the prevalence of 12 month wheeze. A similar ranking of highest centres was found for wheezing severe enough to limit speech to only one or two words at a time between breaths, but for Š 4 attacks of wheezing the ranking of highest centres was similar to 12 month wheeze.

Correlations between prevalence and severity were also examined. However, because the severity questions are not independent of the 12 month wheeze question the correlations were assessed between the proportion of the population wheezing in the last 12 months and the proportion of the population of wheezers in that centre answering positively to questions on severity in the last 12 months. The correlations examining relative severity were: four or more attacks of wheezing ( $\mathrm{r}=0.25, \mathrm{p}=0.0016)$; sleep disturbance due to wheezing on one or more nights per week $(r=-0.097, p=0.23)$; and wheezing severe enough to limit speech to only one or two words at a time between breaths $(\mathrm{r}=0.012, \mathrm{p}=0.89)$, indicating that the proportion of wheezers with severe symptoms changed little with increasing prevalence of wheeze.

Every country had some children who reported having "asthma" at some time in their lives, but the range in prevalence was very large, ranging from 1.6-3.0\% in Albania, Estonia, Ethiopia, Indonesia, Iran, Poland, Russia, South Korea and Uzbekistan to 20.7-28.2\% in Australia, New Zealand, Oman, Peru, Singapore and the UK. The correlation between the proportion of the population wheezing in the last 12 months and the proportion of the population reporting "asthma ever" was $r=0.76(p<0.0001)$. In some countries the reporting of 12 month wheeze was much higher than the reporting of "asthma ever" (e.g. Ethiopia $6.2 \%, 2.5 \%$; Iran 10.9\%, 2.7\%; Brazil 22.7\%, 14.9\%; Canada 28.1\%, 16.5\%; Germany 13.8\%, 5.7\%; "wheeze", "asthma", respectively), whereas in other countries there was much more "asthma" than "wheeze" (e.g. China 4.2\%, $6.1 \%$; Nigeria $10.7 \%, 18.4 \%$; Japan $13.4 \%, 18.9 \%$; Singapore $9.7 \%, 20.9 \%$; "wheeze", "asthma", respectively).

Exercise wheeze in the last 12 months was reported more frequently than wheeze in the last 12 months in all 

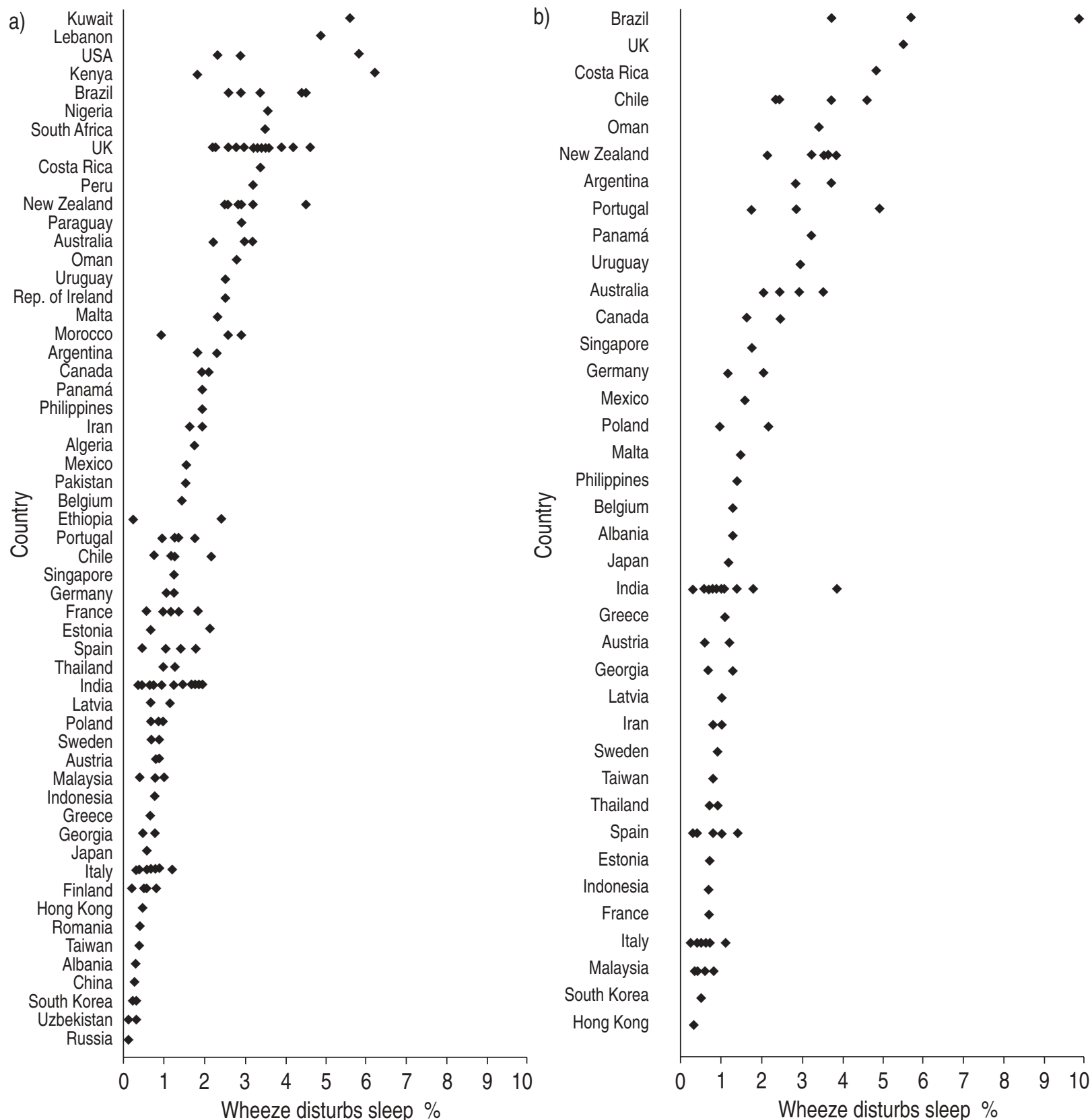

Fig. 2. - Ranking of participating countries for the percentage who answered positively to the question "In the last 12 months, how often, on average, has your (child's) sleep been disturbed due to wheezing?: one or more nights per week" for: a) the 13-14 yr olds; and b) the 6-7 yr olds.

countries except for Greece, Mexico, Panama, Paraguay, Republic of Ireland, the UK and Uruguay, with a correlation of $r=0.83(p<0.0001)$ between these variables. The proportion of children reporting wheezing with exercise in the last 12 months ranged from $2.3 \%$ in Indonesia to $43.4 \%$ in Nigeria.

Dry night cough in the last 12 months was reported more frequently than 12 month wheeze in all countries except for Australia, New Zealand and Sweden, with a correlation of $r=0.81(p<0.0001)$ between these variables. The proportion of children reporting dry night cough in the last 12 months ranged from $4.0 \%$ in Indonesia to $42.3 \%$ in the UK.

The proportion of females and males studied varied among the centres, from 34.1 to $69.9 \%$ males. The pattern of responses to questions between sexes was not consistent. On average females showed a higher prevalence than males for all questions except for reported "asthma ever", where males were higher than females. However, there was a significant interaction with country $(p<0.0001)$, with many countries having a greater proportion of positive responses in males than in females.

6-7 yr old group. The prevalence rates for asthma symptoms are presented by centre and country in table 2 . Eighteen countries were represented by more than one centre. The geographic distribution of prevalence is illustrated, by centre on a world map (fig. 3), for positive answers to the question "Has your child had wheezing or whistling in the chest in the last 12 months?". There was more than 
Table 2. - Summary of parent-completed wheezing questionnaire (written) data, 6-7 yr-old age group

\begin{tabular}{|c|c|c|c|c|c|c|c|c|}
\hline \multirow[t]{2}{*}{ Centre $^{+}$} & \multicolumn{6}{|c|}{ 12-month prevalence } & \multirow[b]{2}{*}{$\begin{array}{l}\text { Ever had } \\
\text { asthma }\end{array}$} & \multirow[b]{2}{*}{$\mathrm{n}$} \\
\hline & Wheeze & Š4 Attacks & $\begin{array}{c}\text { Wheeze } \\
\text { disturbs } \\
\text { sleep }\end{array}$ & $\begin{array}{l}\text { Severe wheeze } \\
\text { limiting speech }\end{array}$ & $\begin{array}{l}\text { Exercise } \\
\text { wheeze }\end{array}$ & $\begin{array}{l}\text { Night } \\
\text { cough }\end{array}$ & & \\
\hline \multicolumn{9}{|l|}{ Asia-Pacific } \\
\hline \multicolumn{9}{|l|}{$\begin{array}{l}\text { Hong Kong } \\
\text { Hong Kong }\end{array}$} \\
\hline & 9.1 & 2.3 & 0.3 & 1.1 & 6.9 & 21.7 & 7.7 & 3618 \\
\hline \multicolumn{9}{|l|}{ Japan } \\
\hline $\begin{array}{l}\text { Fukuoka } \\
\text { Malaysia }\end{array}$ & $\begin{array}{r}17.3 \\
6.1\end{array}$ & $\begin{array}{l}5.4 \\
1.4\end{array}$ & $\begin{array}{l}1.2 \\
0.5\end{array}$ & $\begin{array}{l}1.8 \\
1.1\end{array}$ & $\begin{array}{l}5.3 \\
4.3\end{array}$ & $\begin{array}{r}9.5 \\
16.2\end{array}$ & $\begin{array}{l}18.2 \\
10.4\end{array}$ & $\begin{array}{r}2900 \\
15285\end{array}$ \\
\hline Alor Setar & 6.2 & 1.6 & 0.8 & $\begin{array}{l}1.1 \\
1.4\end{array}$ & 4.7 & 15.8 & $\begin{array}{l}10.4 \\
13.4\end{array}$ & 2978 \\
\hline Ipohe $^{\mathrm{e}}$ & 6.3 & 1.2 & 0.3 & 1.0 & 4.6 & 12.6 & 10.3 & 2506 \\
\hline Klang Valley & 7.8 & 2.1 & 0.6 & 1.4 & 4.9 & 17.9 & 11.1 & 3109 \\
\hline Kota Bharu & 5.6 & 1.1 & 0.4 & 1.0 & 4.0 & 21.0 & 10.4 & 3819 \\
\hline \multirow{2}{*}{\multicolumn{9}{|c|}{ Philippines }} \\
\hline & & & & & & & & \\
\hline Metro Manilla & 11.3 & 1.4 & 1.4 & 2.4 & 6.7 & 30.6 & 16.4 & 3558 \\
\hline Singapore & & & & & & & & \\
\hline Singapore & 15.7 & 3.5 & 1.8 & 1.9 & 8.2 & 15.0 & 18.5 & 2353 \\
\hline South Korea & 13.3 & 1.7 & 0.5 & 2.0 & 4.4 & 17.4 & 8.5 & 8109 \\
\hline Provincial Korea & 12.4 & 1.9 & 0.5 & 2.0 & 4.5 & 17.4 & 8.3 & 5527 \\
\hline Seoula $^{\mathrm{a}}$ & 15.1 & 1.5 & 0.5 & 2.0 & 4.1 & 17.3 & 9.0 & 2582 \\
\hline Taiwan & & & & & & & & \\
\hline Taipei ${ }^{a}$ & 9.6 & 2.9 & 0.8 & 1.2 & 6.0 & 17.0 & 12.7 & 4806 \\
\hline Thailand & 8.2 & 2.4 & 0.8 & 1.3 & 3.9 & 18.4 & 6.7 & 7457 \\
\hline Bangkok & 11.0 & 3.4 & 0.9 & 1.7 & 5.1 & 22.8 & 9.3 & 3629 \\
\hline Chiang Mai & 5.5 & 1.5 & 0.7 & 0.9 & 2.8 & 14.2 & 4.2 & 3828 \\
\hline Regional total & 9.6 & 2.2 & 0.7 & 1.5 & 5.0 & 17.6 & 10.7 & 49476 \\
\hline Eastern Mediterranea & & & & & & & & \\
\hline Iran & 5.4 & 1.0 & 0.9 & 0.9 & 1.8 & 5.9 & 3.0 & 5469 \\
\hline Rasht & 5.3 & 1.0 & 1.0 & 1.1 & 1.8 & 5.7 & 4.1 & 3013 \\
\hline Tehran & 5.5 & 1.0 & 0.8 & 0.8 & 1.7 & 6.0 & 1.7 & 2456 \\
\hline Malta & & & & & & & & \\
\hline Malta & 8.8 & 1.7 & 1.5 & 1.2 & 4.1 & 19.1 & 7.5 & 3493 \\
\hline Oman & & & & & & & & \\
\hline Al-Khod & 7.1 & 2.5 & 3.5 & 3.2 & 6.9 & 19.6 & 10.5 & 3891 \\
\hline Regional total & 6.8 & 1.7 & 1.8 & 1.7 & 4.0 & 13.6 & 6.5 & 12853 \\
\hline Latin America & & & & & & & & \\
\hline Argentina & 16.4 & 3.8 & 3.4 & 4.3 & 6.2 & 39.5 & 5.3 & 6012 \\
\hline Buenos Aires & 15.4 & 3.2 & 2.9 & 3.2 & 4.9 & 37.5 & 4.1 & 3005 \\
\hline Rosario & 17.3 & 4.4 & 3.8 & 5.4 & 7.5 & 41.5 & 6.5 & 3007 \\
\hline Brazil & 23.3 & 5.3 & 5.8 & 4.4 & 7.5 & 34.2 & 13.1 & 7261 \\
\hline Porto Alegre & 23.5 & 5.0 & 5.8 & 4.0 & 6.9 & 33.8 & 16.8 & 2846 \\
\hline Recife $^{f}$ & 27.2 & 8.3 & 10.0 & 9.6 & 13.0 & 34.8 & 20.4 & 1410 \\
\hline São Paulo & 21.3 & 4.1 & 3.8 & 2.3 & 5.5 & 34.2 & 6.1 & 3005 \\
\hline Chile & 17.9 & 2.9 & 3.5 & 3.0 & 11.0 & 25.7 & 12.1 & 10838 \\
\hline Central Santiago & 16.5 & 1.6 & 2.4 & 1.9 & 8.0 & 23.7 & 9.7 & 1458 \\
\hline Punta Arenas & 17.1 & 2.1 & 2.5 & 2.6 & 10.1 & 29.5 & 10.4 & 3060 \\
\hline South Santiago & 16.9 & 2.6 & 3.8 & 3.6 & 9.3 & 24.0 & 10.5 & 3182 \\
\hline Valdivia & 20.5 & 4.5 & 4.7 & 3.3 & 15.2 & 24.7 & 16.5 & 3138 \\
\hline Costa Rica & & & & & & & & \\
\hline Costa Rica & 32.1 & 7.3 & 4.9 & 11.3 & 16.1 & 38.3 & 26.9 & 2942 \\
\hline Mexico & & & & & & & & \\
\hline Cuernavaca & 8.6 & 1.3 & 1.6 & 2.8 & 4.0 & 19.9 & 5.1 & 3097 \\
\hline Panama & & & & & & & & \\
\hline David-Panamáe & 23.5 & 4.1 & 3.3 & 6.2 & 12.3 & 29.3 & 19.3 & 3043 \\
\hline $\begin{array}{l}\text { Uruguay } \\
\text { Montevideob,e }\end{array}$ & 180 & & & & & & & \\
\hline Regional total & 19.6 & $\begin{array}{l}4.6 \\
\mathbf{4 . 0}\end{array}$ & $\begin{array}{l}3.0 \\
3.8\end{array}$ & $\begin{array}{l}3.3 \\
4.5\end{array}$ & 7.0 & 26.4 & 12.0 & $\begin{array}{r}3071 \\
\mathbf{3 6 2 6 4}\end{array}$ \\
\hline North America & 19.0 & & & & 9.1 & 30.0 & 12.4 & 50204 \\
\hline Canada & 17.6 & 5.5 & 2.2 & 3.0 & 9.6 & 25.1 & 14.7 & 5755 \\
\hline Hamilton & 20.1 & 6.2 & 2.5 & 3.3 & 10.7 & 27.7 & 17.2 & 3337 \\
\hline Saskatoon & 14.1 & 4.5 & 1.7 & 2.6 & 8.1 & 21.5 & 11.2 & 2418 \\
\hline Regional total & 17.6 & 5.5 & 2.2 & 3.0 & 9.6 & 25.1 & 14.7 & 5755 \\
\hline Northern and Eastern & & & & & & & & \\
\hline Albania & & & & & & & & \\
\hline Tiranë & 7.6 & 1.0 & 1.3 & 2.4 & 4.8 & 9.3 & 3.1 & 2981 \\
\hline Estonia & & & & & & & & \\
\hline Tallinn & 9.3 & 1.5 & 0.7 & 0.7 & 1.6 & 11.7 & 1.4 & 3070 \\
\hline Georgia & 7.6 & 1.5 & 1.0 & 1.3 & 3.3 & 7.0 & 3.1 & 6770 \\
\hline Kutaisi & 9.3 & 2.0 & 1.3 & 1.5 & 4.5 & 8.7 & 4.5 & 3356 \\
\hline Tbilisi & 5.9 & 1.0 & 0.7 & 1.1 & 2.1 & 5.4 & 1.8 & 3414 \\
\hline Latvia & & & & & & & & \\
\hline Riga & 7.3 & 1.3 & 1.0 & 0.9 & 2.2 & 7.8 & 1.6 & 3003 \\
\hline Poland & 10.9 & 3.0 & 1.5 & 2.1 & 3.8 & 18.5 & 2.5 & 4974 \\
\hline Krakow (1995) & 14.3 & 3.8 & 2.2 & 2.4 & 4.9 & 25.1 & 4.1 & 2264 \\
\hline Poznan & 8.1 & 2.3 & 1.0 & 1.7 & 2.9 & 13.0 & 1.3 & 2710 \\
\hline
\end{tabular}


Table 2. - continued.

\begin{tabular}{|c|c|c|c|c|c|c|c|c|}
\hline \multirow[t]{2}{*}{ Centre $^{+}$} & \multicolumn{6}{|c|}{ 12-month prevalence } & \multirow[b]{2}{*}{$\begin{array}{c}\text { Ever had } \\
\text { asthma }\end{array}$} & \multirow[b]{2}{*}{$\mathrm{n}$} \\
\hline & Wheeze & Š4 Attacks & $\begin{array}{c}\text { Wheeze } \\
\text { disturbs } \\
\text { sleep }\end{array}$ & $\begin{array}{l}\text { Severe wheeze } \\
\text { limiting speech }\end{array}$ & $\begin{array}{l}\text { Exercise } \\
\text { wheeze }\end{array}$ & $\begin{array}{l}\text { Night } \\
\text { cough }\end{array}$ & & \\
\hline \multicolumn{9}{|l|}{ Sweden } \\
\hline Stockholm/Uppsala & 10.4 & 3.3 & 0.9 & 1.5 & 6.2 & 14.6 & 8.0 & 3029 \\
\hline Regional total & 8.8 & 2.0 & 1.1 & 1.5 & 3.6 & 11.4 & 3.2 & 23827 \\
\hline \multicolumn{9}{|l|}{ Oceania } \\
\hline Australia & 24.6 & 8.7 & 2.8 & 3.9 & 14.8 & 29.8 & 27.1 & 10899 \\
\hline Adelaide & 26.2 & 9.2 & 3.6 & 4.4 & 15.5 & 30.4 & 27.5 & 3063 \\
\hline Melbourne & 27.2 & 9.1 & 2.5 & 4.2 & 15.8 & 32.2 & 28.6 & 2840 \\
\hline Perth & 22.0 & 9.0 & 3.0 & 4.4 & 15.3 & 29.7 & 28.4 & 2192 \\
\hline Sydney & 22.3 & 7.5 & 2.1 & 2.6 & 12.5 & 26.7 & 24.4 & 2804 \\
\hline New Zealand & 24.5 & 9.0 & 3.5 & 5.1 & 16.5 & 29.2 & 26.5 & 18569 \\
\hline Auckland & 22.5 & 7.9 & 3.7 & 5.3 & 14.8 & 27.5 & 23.8 & 3526 \\
\hline Bay of Plenty & 24.0 & 8.2 & 3.6 & 4.8 & 15.9 & 28.9 & 25.7 & 2681 \\
\hline Christchurch & 27.2 & 10.2 & 3.9 & 5.0 & 19.2 & 32.2 & 28.4 & 3318 \\
\hline Hawke's Bay & 27.0 & 9.9 & 3.7 & 5.6 & 17.7 & 30.7 & 28.3 & 3338 \\
\hline Nelson & 18.7 & 7.1 & 2.2 & 4.6 & 13.2 & 21.1 & 17.6 & 1868 \\
\hline Wellington & 25.1 & 9.8 & 3.3 & 4.7 & 16.9 & 31.1 & 30.8 & 3838 \\
\hline Regional total & 24.6 & 8.9 & 3.2 & 4.6 & 15.9 & 29.4 & 26.8 & 29468 \\
\hline \multicolumn{9}{|l|}{ South-east Asia } \\
\hline India & 5.6 & 1.5 & 1.2 & 1.9 & 3.6 & 12.3 & 3.7 & 31697 \\
\hline Akola & 0.8 & 0.6 & 0.3 & 0.6 & 1.0 & 3.3 & 1.3 & 2030 \\
\hline Bombay (16) & 3.8 & 1.3 & 1.0 & 1.6 & 3.0 & 12.6 & 3.8 & 3967 \\
\hline Bombay (17) & 5.4 & 1.0 & 1.0 & 1.3 & 4.3 & 16.1 & 2.9 & 1148 \\
\hline Bombay (18) & 1.8 & 0.7 & 0.6 & 0.7 & 1.8 & 8.3 & 2.3 & 3568 \\
\hline Borivali & 5.2 & 2.0 & 1.4 & 1.7 & 3.1 & 12.3 & 3.4 & 1672 \\
\hline Chandigarh & 5.4 & 1.9 & 1.8 & 2.8 & 3.8 & 10.7 & 2.8 & 2891 \\
\hline Jodhpur & 3.5 & 1.3 & 1.0 & 1.4 & 2.9 & 13.6 & 4.1 & 1104 \\
\hline Kottayam & 24.6 & 4.7 & 3.9 & 7.5 & 13.3 & 27.0 & 14.4 & 2156 \\
\hline Madras (2) & 7.2 & 2.1 & 0.9 & 1.4 & 2.5 & 16.4 & 1.4 & 1466 \\
\hline Madras (3) & 8.5 & 2.4 & 1.4 & 2.5 & 3.8 & 15.4 & 2.2 & 2491 \\
\hline New Delhi & 6.9 & 1.4 & 0.7 & 1.6 & 4.1 & 14.6 & 3.7 & 2938 \\
\hline Neyveli & 1.5 & 0.1 & 0.3 & 0.3 & 1.4 & 8.1 & 1.0 & 1498 \\
\hline Orissa & 4.1 & 1.4 & 1.1 & 2.2 & 3.8 & 8.7 & 3.8 & 1520 \\
\hline Pune & 2.3 & 1.0 & 0.8 & 1.3 & 2.5 & 9.5 & 4.2 & 3248 \\
\hline Regional total & 5.6 & 1.5 & 1.2 & 1.9 & 3.6 & 12.3 & 3.7 & 31697 \\
\hline \multicolumn{9}{|l|}{ Western Europe } \\
\hline Austria & 8.9 & 1.6 & 1.0 & 2.0 & 5.3 & 14.2 & 3.9 & 5787 \\
\hline Salzburg & 9.7 & 1.8 & 1.2 & 2.5 & 5.7 & 16.4 & 4.2 & 3658 \\
\hline Urfahr-Ümgebung & 7.7 & 1.2 & 0.6 & 1.1 & 4.6 & 10.4 & 3.3 & 2129 \\
\hline Belgium & & & & & & & & \\
\hline Antwerp & 7.3 & 2.0 & 1.3 & 1.2 & 3.6 & 14.8 & 4.2 & 6533 \\
\hline France & & & & & & & & \\
\hline Pessac ${ }^{a}$ & 8.1 & 2.6 & 0.7 & 0.7 & 3.3 & 16.4 & 9.3 & 3202 \\
\hline Germany & 8.5 & 1.9 & 1.7 & 2.0 & 5.1 & 11.1 & 3.6 & 6592 \\
\hline Greifswald & 7.2 & 1.4 & 1.2 & 1.8 & 3.8 & 8.0 & 2.9 & 2853 \\
\hline Münster & 9.6 & 2.2 & 2.1 & 2.1 & 6.0 & 13.5 & 4.1 & 3739 \\
\hline Greece & & & & & & & & \\
\hline Athens & 7.6 & 1.4 & 1.1 & 0.7 & 2.4 & 12.8 & 5.4 & 1654 \\
\hline Italy & 7.3 & 1.4 & 0.6 & 1.1 & 1.8 & 17.9 & 8.6 & 20815 \\
\hline Cremona & 5.7 & 0.8 & 0.7 & 0.6 & 1.0 & 12.9 & 4.6 & 1392 \\
\hline Emilia-Romagna & 7.4 & 1.4 & 0.7 & 1.1 & 1.5 & 16.1 & 6.9 & 4472 \\
\hline Empoli & 8.7 & 0.8 & 0.5 & 0.8 & 1.0 & 19.2 & 7.8 & 1434 \\
\hline Firenze & 9.9 & 1.9 & 1.1 & 1.3 & 2.5 & 19.8 & 8.8 & 1138 \\
\hline Milano & 7.0 & 1.5 & 0.6 & 1.3 & 2.2 & 22.6 & 9.1 & 3616 \\
\hline Roma & 7.2 & 1.5 & 0.5 & 1.1 & 1.8 & 18.2 & 10.3 & 4027 \\
\hline Torino & 6.4 & 1.2 & 0.5 & 1.4 & 1.4 & 17.7 & 7.6 & 1429 \\
\hline Verona & 7.4 & 1.7 & 0.4 & 1.1 & 3.2 & 16.1 & 11.7 & 2076 \\
\hline Viterbo $^{d}$ & 6.7 & 1.5 & 0.2 & 1.0 & 1.5 & 16.0 & 8.4 & 1231 \\
\hline Portugal & 13.2 & 3.8 & 3.4 & 4.5 & 7.9 & 28.3 & 11.0 & 5129 \\
\hline Funchal & 14.7 & 5.2 & 5.0 & 7.2 & 11.4 & 34.9 & 17.5 & 1797 \\
\hline Lisbon & 13.1 & 3.5 & 2.9 & 3.1 & 6.7 & 26.3 & 8.3 & 2143 \\
\hline Portimao & 11.0 & 2.1 & 1.8 & 3.1 & 4.6 & 22.0 & 6.2 & 1189 \\
\hline Spain & 6.2 & 1.4 & 0.8 & 1.0 & 2.9 & 12.2 & 6.2 & 16884 \\
\hline Bilbao & 8.0 & 2.1 & 1.0 & 1.3 & 4.7 & 15.9 & 10.1 & 3019 \\
\hline Cartagena $^{b}$ & 8.4 & 1.6 & 1.4 & 1.5 & 3.1 & 16.0 & 6.2 & 3335 \\
\hline Castellón & 4.7 & 0.9 & 0.8 & 0.8 & 2.1 & 8.5 & 4.3 & 3594 \\
\hline Pamplona & 3.5 & 0.7 & 0.3 & 0.4 & 1.5 & 9.7 & 4.3 & 2996 \\
\hline Valencia & 6.2 & 1.5 & 0.4 & 1.0 & 2.9 & 11.5 & 6.2 & 3940 \\
\hline UK & & & & & & & & \\
\hline Sunderland & 18.4 & 6.8 & 5.6 & 3.4 & 13.5 & 28.1 & 22.9 & 1864 \\
\hline Regional total & 8.1 & 1.9 & 1.2 & 1.5 & 3.7 & 16.1 & 7.2 & 68460 \\
\hline Global total & 11.8 & 3.1 & 1.8 & 2.4 & 6.2 & 19.1 & 10.2 & 257800 \\
\hline
\end{tabular}

All data are presented as percentages of the study children. a: data re-coded to eliminate inconsistent responses; b: response rate $<70 \%$; : $<10$ schools if Š 10 schools in sampling frame; ${ }^{\mathrm{d}}:$ 13-14 yr age group not included in this paper; ${ }^{\mathrm{e}}:$ single data entry; f: no date of interview or date of birth on questionnaire; g: no eczema questionnaire. +: numbers in brackets indicate International Study of Asthma and Allergies in Childhood (ISAAC) registration number for that centre, as given by the national coordinator. 


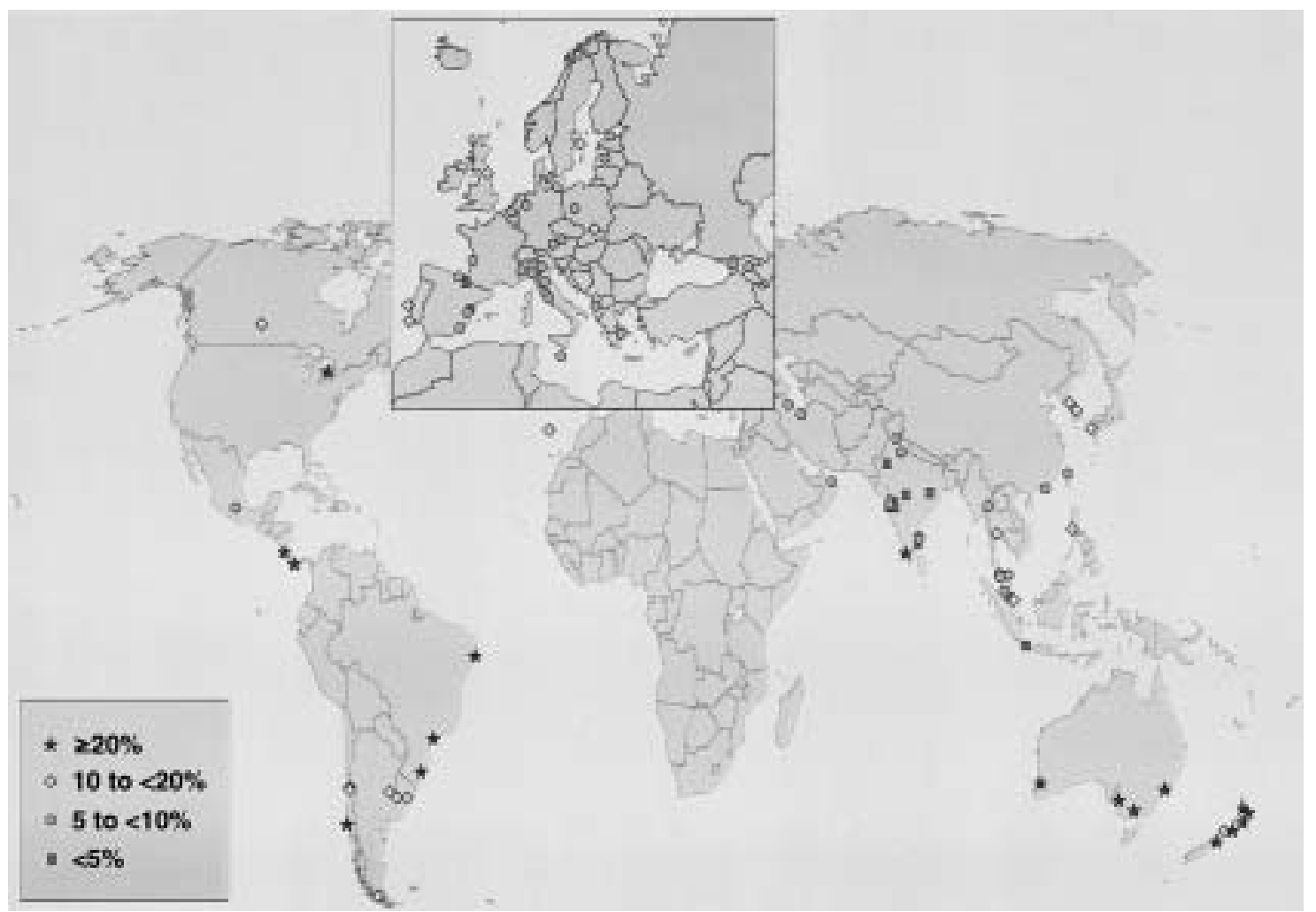

Fig. 3. - World map for the 6-7 yr old age group, showing the percentage of parents who answered "yes" to the written question "Has your child had wheezing or whistling in the chest in the last 12 months?". Each point represents the prevalence for one collaborating centre, with distinct colour symbols used for different prevalence ranges.

a fivefold difference in prevalence between countries (4.1-32.1\%), with lowest rates in India, Indonesia, Iran and Malaysia and highest rates in Australia, Brazil, Costa Rica, New Zealand and Panama. Some centres in countries with extreme values in the older age group did not provide data for the younger age group.

The between-country variation (38 countries) was greater than the within-country variation (18 countries with $>1$ centre) for all questions: 12 month wheeze was 1.1 times larger; four or more attacks of wheezing in the last 12 months 1.8; waking one or more nights a week 2.0; wheezing severe enough to limit speech to only one or two words at a time between breaths 1.5; wheeze with exercise 1.7; dry cough at night 1.6 , and reported asthma ever 2.0 times larger.

Both age groups were studied in 90 centres and the correlations between the two age groups for the different symptoms ranged from $r=0.61$ to $r=0.89$. The question $a b-$ out wheezing in the last 12 months is illustrated in figure 4 $(r=0.71, p<0.0001)$, with 57 centres having a lower prevalence in the younger age group and 33 centres having a higher prevalence in the younger age group. The 6-7 yr age group also had lower prevalences than the 13-14 yr age group for all the other symptoms except for any sleep disturbance due to wheezing.

The ranking of countries for sleep disturbance due to wheezing on one or more nights per week is illustrated in figure 2 . The countries with the highest prevalence of positive answers to this question were Brazil, Costa Rica and the UK, and the lowest were Hong Kong, Malaysia and South Korea. A similar pattern was found for wheezing severe enough to limit speech to only one or two words at

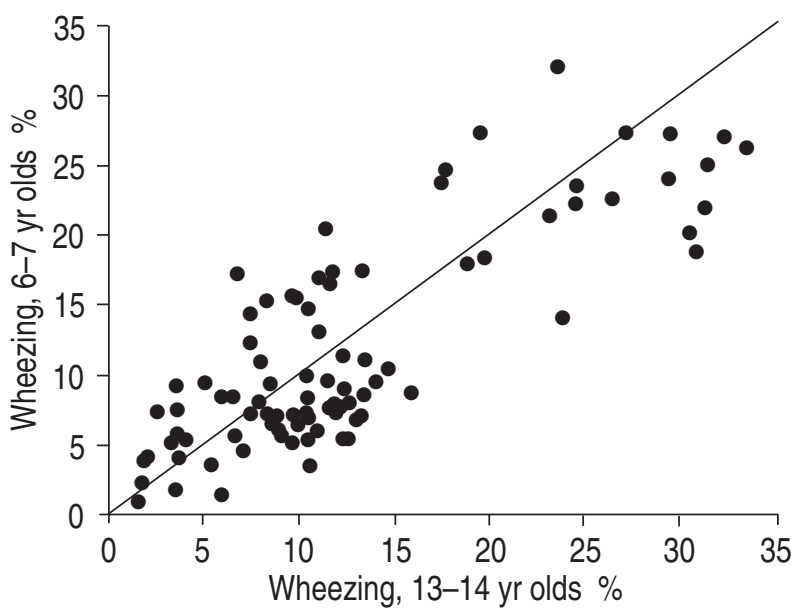

Fig. 4. - Scatter plots for centres for the percentage of children who have had "wheezing or whistling in the chest in the last 12 months". The x-axis shows self-reported wheezing in 13-14 yr olds and the y-axis wheezing reported by parents in 6-7 yr olds. The line of identity is shown.

a time between breaths, but for $\breve{S} 4$ attacks of wheezing the pattern was similar to 12 month wheeze.

Correlations done between these variables, examining relative severity (as described above for the 13-14 yr age group), were similar to the older age group: four or more attacks of wheezing $(\mathrm{r}=0.12, \mathrm{p}=0.25)$; sleep disturbance due to wheezing on one or more nights per week $(r=-0.04$, $\mathrm{p}=0.74$ ); and wheezing severe enough to limit speech to only one or two words at a time between breaths $(\mathrm{r}=-0.13$, $\mathrm{p}=0.21$ ), indicating that the proportion of wheezy children 
with severe asthma symptoms changed little with increasing prevalence of wheeze.

Some parents from all countries reported "asthma ever" in their children, but the range in prevalence was very large, ranging from $1.4-4.2 \%$ in Albania, Austria, Belgium, Estonia, Germany, India, Iran, Latvia, Poland and Georgia to 26.5-27.1\% in Australia, Costa Rica and New Zealand. The correlation between the proportion of the population wheezing in the last 12 months and the proportion of the population reporting "asthma ever" was $r=0.73$ $(p<0.0001)$. In some countries the reporting of 12 month wheeze was much higher than the reporting of "asthma ever" (e.g. Brazil 23.3\%, 13.1\%; Estonia 9.3\%, 1.4\%; Iran 5.4\%, 3.0\%; "wheeze", "asthma", respectively), whereas in other countries there was more "asthma ever" than 12 month wheeze (e.g. Philippines 11.3\%, 16.4\%; Oman 7.1\%, 10.5\%; "wheeze", "asthma", respectively).

Exercise wheeze in the last 12 months was reported less frequently than 12 month wheeze in all countries, with a correlation of $r=0.84(p<0.0001)$ between these variables. The proportion of reported wheezing with exercise in the last 12 months ranged from $1.6 \%$ in Estonia to $16.5 \%$ in New Zealand. In contrast to the lower rates of exercise wheeze in this age group, dry night cough in the last 12 months was reported more frequently than 12 month wheeze in all countries except for Japan, Singapore and Georgia, with a correlation of $\mathrm{r}=0.82(\mathrm{p}<0.0001)$ between these variables. The proportion of parents of children reporting dry night cough in the last 12 months ranged from $5.9 \%$ in Iran to $39.5 \%$ in Argentina.

The proportion of females and males studied varied among the centres, from 40.0 to $58.6 \%$ males, and a clear pattern emerged. Males showed a significantly higher prevalence than females in this age group $(\mathrm{p}<0.001)$ for 12 month wheezing (1:0.81), four or more attacks of wheezing per week (1:0.76), waking on one or more nights a week (1:0.92), wheezing severe enough to limit speech to only one or two words at a time between breaths (1:0.78), wheeze with exercise (1:0.79) and dry cough at night (1: 0.92). There was no significant interaction with country.

\section{Video questionnaire}

Ninety-nine centres in 42 countries undertook the video questionnaire. The prevalence rates for video symptoms of asthma are presented by centre and country in table 3 . The geographic distribution is illustrated, by centre on a world map (fig. 5), for positive answers to the scene of wheezing

Table 3. - Summary of self-completed wheezing questionnaire (video) data, 13-14 yr-old age group

\begin{tabular}{|c|c|c|c|c|c|c|c|}
\hline \multirow[t]{2}{*}{ Centre } & \multirow[t]{2}{*}{ Video } & \multicolumn{5}{|c|}{ Prevalence in the last year } & \multirow[t]{2}{*}{$\mathrm{n}$} \\
\hline & & Wheeze & $\begin{array}{c}\text { Exercise } \\
\text { wheeze }\end{array}$ & $\begin{array}{c}\text { Night } \\
\text { wheeze }\end{array}$ & $\begin{array}{l}\text { Night } \\
\text { cough }\end{array}$ & $\begin{array}{c}\text { Severe } \\
\text { wheeze }\end{array}$ & \\
\hline \multicolumn{8}{|l|}{ Africa } \\
\hline Kenya & & 11.4 & 15.0 & 6.7 & 16.2 & 7.5 & 6257 \\
\hline Eldoret & I & 3.5 & 5.0 & 1.4 & 0.0 & 1.4 & 3016 \\
\hline Nairobi & I & 18.7 & 24.3 & 11.7 & 31.2 & 13.1 & 3241 \\
\hline \multicolumn{8}{|l|}{ South Africa } \\
\hline Cape Town & I & 6.5 & 11.5 & 3.9 & 11.6 & 5.0 & 5164 \\
\hline Regional total & & 9.2 & 13.4 & 5.4 & 14.1 & 6.3 & 11421 \\
\hline \multicolumn{8}{|l|}{ Asia-Pacific } \\
\hline China & & 2.0 & 5.1 & 0.6 & 4.9 & 1.2 & 18985 \\
\hline Beijing & I & 3.3 & 4.7 & 0.6 & 6.8 & 1.4 & 4165 \\
\hline Chongaing & I & 1.3 & 5.2 & 0.4 & 3.3 & 0.8 & 4289 \\
\hline Guangzhou & I & 2.0 & 6.9 & 0.7 & 5.4 & 1.1 & 3854 \\
\hline Shanghai & I & 1.6 & 3.5 & 0.9 & 1.8 & 1.1 & 3470 \\
\hline Wulumuqi & I & 1.8 & 5.4 & 0.4 & 7.4 & 1.6 & 3207 \\
\hline \multicolumn{8}{|l|}{ Hong Kong } \\
\hline Hong Kong & I & 10.1 & 15.3 & 3.8 & 24.6 & 6.9 & 4665 \\
\hline \multicolumn{8}{|l|}{ Indonesia } \\
\hline Bandung & I & 1.3 & 2.8 & 0.6 & 1.8 & 0.8 & 2246 \\
\hline \multicolumn{8}{|l|}{ Japan } \\
\hline Fukuoka & I & 10.2 & 16.5 & 3.7 & 7.2 & 5.3 & 2820 \\
\hline Malaysia & & 5.9 & 9.2 & 2.5 & 8.4 & 3.6 & 15571 \\
\hline Alor Setar & I & 5.3 & 8.6 & 2.1 & 8.6 & 2.7 & 3295 \\
\hline Ipoh & I & 6.5 & 10.8 & 3.2 & 9.9 & 5.8 & 3271 \\
\hline Klang Valley & I & 6.9 & 9.7 & 2.7 & 9.0 & 3.7 & 5966 \\
\hline Kota Bharu & I & 3.9 & 7.0 & 1.8 & 5.2 & 2.2 & 3039 \\
\hline \multicolumn{8}{|l|}{ Philippines } \\
\hline Metro Manilla & I & 9.6 & 12.6 & 3.9 & 14.6 & 4.9 & 3207 \\
\hline \multicolumn{8}{|l|}{ Singapore } \\
\hline Singapore & I & 9.9 & 8.1 & 3.2 & 9.0 & 5.0 & 4189 \\
\hline South Korea & & 3.7 & 6.7 & 0.5 & 4.0 & 1.9 & 9932 \\
\hline Provincial Korea* & I & 3.1 & 6.0 & 0.5 & 3.3 & 1.6 & 6972 \\
\hline Seoul* & I & 5.2 & 8.2 & 0.7 & 5.8 & 2.6 & 2960 \\
\hline \multicolumn{8}{|l|}{ Taiwan } \\
\hline Taipei* & I & 4.6 & 6.0 & 1.8 & 3.4 & 2.8 & 4211 \\
\hline Thailand & & 6.9 & 10.1 & 2.1 & 12.3 & 3.8 & 7629 \\
\hline Bangkok & I & 8.0 & 12.7 & 2.7 & 14.4 & 4.6 & 3705 \\
\hline Chiang Mai & I & 6.0 & 7.7 & 1.7 & 10.2 & 3.1 & 3924 \\
\hline Regional total & & 5.3 & 8.3 & 1.8 & 8.1 & 3.1 & 73455 \\
\hline \multicolumn{8}{|l|}{ Eastern Mediterranean } \\
\hline \multicolumn{8}{|l|}{ Iran } \\
\hline Rasht & I & 3.1 & 6.1 & 1.1 & 6.0 & 2.1 & 3175 \\
\hline Kuwait & & & & & & & \\
\hline Kuwait & E & 13.3 & 14.6 & 9.4 & 9.7 & 9.1 & 1033 \\
\hline Lebanon & & & & & & & \\
\hline Beirut & I & 4.9 & 8.4 & 3.6 & 7.4 & 3.2 & 2985 \\
\hline
\end{tabular}


Table 3. - continued.

\begin{tabular}{|c|c|c|c|c|c|c|c|}
\hline \multirow[t]{2}{*}{ Centre } & \multirow[t]{2}{*}{ Video } & \multicolumn{5}{|c|}{ Prevalence in the last year } & \multirow[t]{2}{*}{$\mathrm{n}$} \\
\hline & & Wheeze & $\begin{array}{l}\text { Exercise } \\
\text { wheeze }\end{array}$ & $\begin{array}{c}\text { Night } \\
\text { wheeze }\end{array}$ & $\begin{array}{l}\text { Night } \\
\text { cough }\end{array}$ & $\begin{array}{c}\text { Severe } \\
\text { wheeze }\end{array}$ & \\
\hline \multicolumn{8}{|l|}{ Malta } \\
\hline Malta & I & 8.8 & 11.1 & 4.0 & 17.8 & 5.5 & 4173 \\
\hline Morocco & & 7.5 & 9.5 & 3.8 & 11.7 & 5.6 & 9350 \\
\hline Casablanca & E & 10.6 & 13.0 & 5.5 & 14.5 & 8.3 & 3182 \\
\hline Marrakech & $\mathrm{E}$ & 5.6 & 7.7 & 2.5 & 10.6 & 3.8 & 2899 \\
\hline Rabat & $\mathrm{I}$ & 6.2 & 7.6 & 3.4 & 9.9 & 4.6 & 3269 \\
\hline \multicolumn{8}{|l|}{ Pakistan } \\
\hline Karachi & I & 7.9 & 6.3 & 3.1 & 6.1 & 4.4 & 1829 \\
\hline $\begin{array}{l}\text { Regional total } \\
\text { Latin America }\end{array}$ & & 7.1 & 9.1 & 3.6 & 10.9 & 4.8 & 22545 \\
\hline Argentina & & 8.3 & 9.4 & 4.7 & 12.8 & 5.5 & 5089 \\
\hline Buenos Aires & I & 7.8 & 9.6 & 4.2 & 11.0 & 5.8 & 2498 \\
\hline Rosario & I & 8.8 & 9.1 & 5.2 & 14.5 & 5.1 & 2591 \\
\hline Chile & & 11.2 & 14.2 & 3.5 & 15.4 & 4.5 & 6303 \\
\hline Punta Arenas & I & 9.5 & 11.7 & 2.7 & 14.7 & 4.4 & 3253 \\
\hline South Santiago & I & 13.0 & 16.9 & 4.4 & 16.2 & 4.5 & 3050 \\
\hline Paraguay & & & & & & & \\
\hline Asunción & I & 10.1 & 14.5 & 5.3 & 12.7 & 6.1 & 2966 \\
\hline${ }_{\text {Lima }}^{\text {Peru }}$ & & & & & & & \\
\hline $\begin{array}{r}\text { Lima } \\
\text { Uruguay }\end{array}$ & I & 18.5 & 21.4 & 6.4 & 16.8 & 8.3 & 3151 \\
\hline Montevideo & I & 15.0 & 17.6 & 5.9 & 19.3 & 11.0 & 3069 \\
\hline $\begin{array}{l}\text { Regional total } \\
\text { North America }\end{array}$ & & 12.0 & 14.7 & 4.9 & 15.1 & 6.5 & 20578 \\
\hline $\begin{array}{l}\text { Canada } \\
\text { Canca }\end{array}$ & & 12.0 & 281 & 65 & & & \\
\hline Hamilton & E & 13.4 & 31.0 & $\begin{array}{l}6.5 \\
7.2\end{array}$ & 21.2 & $\begin{array}{l}8.5 \\
8.6\end{array}$ & $\begin{array}{l}4447 \\
3047\end{array}$ \\
\hline Saskatoon & I & 9.9 & 23.5 & 5.4 & 18.2 & 8.3 & 1900 \\
\hline USA & & & & & & & \\
\hline Seattle* & I & 13.0 & 22.5 & 4.6 & 13.5 & 10.6 & 2140 \\
\hline Regional total & & 12.3 & 26.4 & 5.9 & 18.1 & 9.1 & 7087 \\
\hline Northern and Eastern Europe & & & & & & & \\
\hline Albania & & & & & & & \\
\hline Tiranë & I & 1.0 & 1.3 & 0.4 & 1.6 & 0.6 & 2362 \\
\hline Estonia & & & & & & & \\
\hline Tallinn & I & 2.1 & 5.4 & 1.2 & 6.1 & 0.9 & 3454 \\
\hline Finland & & 5.1 & 8.6 & 1.2 & 12.5 & 2.8 & 11888 \\
\hline Helsinki & I & 5.8 & 9.3 & 1.5 & 14.7 & 2.6 & 2853 \\
\hline Kuopio County & I & 3.8 & 7.5 & 0.9 & 11.1 & 2.6 & 2877 \\
\hline Lapland Area & I & 5.4 & 7.6 & 1.2 & 11.6 & 3.4 & 3074 \\
\hline Turku and Pori County & I & 5.2 & 9.9 & 1.2 & 12.5 & 2.6 & 3084 \\
\hline Latvia & & 1.3 & 2.6 & 1.1 & 2.1 & 0.6 & 5957 \\
\hline Riga & I & 1.3 & 3.2 & 0.7 & 3.3 & 0.6 & 2847 \\
\hline Rural Latvia & I & 1.3 & 1.9 & 1.4 & 1.1 & 0.6 & 3110 \\
\hline Poland & & & & & & & \\
\hline Poznan & I & 3.9 & 8.6 & 1.5 & 8.5 & 2.3 & 3238 \\
\hline Russia & & & & & & & \\
\hline Moscow & E & 1.3 & 1.9 & 0.8 & 1.1 & 0.3 & 3411 \\
\hline Sweden & & 5.2 & 12.0 & 1.6 & 8.2 & 2.9 & 6418 \\
\hline Linköping & E & 5.1 & 10.4 & 1.0 & 7.3 & 2.5 & 3363 \\
\hline Stockholm/Uppsala & E & 5.3 & 13.8 & 2.3 & 9.2 & 3.4 & 3055 \\
\hline Uzbekistan & & 1.3 & 1.4 & 0.6 & 1.3 & 0.5 & 4662 \\
\hline Samarkand & $\mathrm{E}$ & 1.8 & 2.3 & 0.7 & 2.1 & 0.4 & 1758 \\
\hline Tashkent & $\mathrm{E}$ & 1.0 & 0.9 & 0.6 & 0.8 & 0.6 & 2904 \\
\hline Regional total & & 3.2 & 6.2 & 1.1 & 6.7 & 1.7 & 41390 \\
\hline Oceania & & & & & & & \\
\hline Australia & & 17.6 & 27.2 & 11.0 & 18.7 & 11.3 & 12193 \\
\hline Adelaide & E & 19.6 & 29.5 & 11.7 & 20.5 & 12.5 & 2990 \\
\hline Melbourne & $\mathrm{E}$ & 15.5 & 25.8 & 10.5 & 16.9 & 10.8 & 2726 \\
\hline Perth & E & 16.8 & 28.0 & 9.9 & 18.2 & 10.6 & 3641 \\
\hline Sydney & $\mathrm{E}$ & 18.4 & 25.1 & 12.2 & 19.2 & 11.6 & 2836 \\
\hline New Zealand & & 18.4 & 30.3 & 11.7 & 22.8 & 12.4 & 18957 \\
\hline Auckland & E & 16.3 & 28.4 & 11.3 & 20.7 & 11.4 & 3201 \\
\hline Bay of Plenty & $\mathrm{E}$ & 18.6 & 28.4 & 11.4 & 25.2 & 12.8 & 2810 \\
\hline Christchurch & $\mathrm{E}$ & 17.4 & 32.2 & 11.3 & 22.4 & 13.2 & 3172 \\
\hline Hawke's Bay & $\mathrm{E}$ & 19.6 & 29.9 & 12.7 & 22.5 & 9.8 & 3536 \\
\hline Nelson & $\mathrm{E}$ & 19.0 & 32.3 & 10.5 & 23.3 & 11.7 & 1827 \\
\hline Wellington & $\mathrm{E}$ & 19.5 & 31.0 & 12.2 & 23.1 & 14.9 & 4411 \\
\hline Regional total & & 18.1 & 29.1 & 11.4 & 21.2 & 12.0 & 31150 \\
\hline South-east Asia & & & & & & & \\
\hline India & & 2.9 & 5.5 & 2.3 & 3.7 & 2.5 & 30043 \\
\hline Bombay (16) & I & 0.8 & 2.2 & 0.9 & 0.9 & 0.7 & 4178 \\
\hline Bombay (18) & I & 1.4 & 4.1 & 0.8 & 3.4 & 2.3 & 3128 \\
\hline Borivali & I & 2.6 & 5.3 & 1.3 & 3.3 & 2.0 & 3869 \\
\hline Chandigarh & I & 2.8 & 5.8 & 2.6 & 3.0 & 1.9 & 3087 \\
\hline Jodhpur & I & 5.8 & 8.4 & 4.0 & 5.4 & 4.4 & 1063 \\
\hline Madras (2) & I & 4.3 & 5.7 & 2.9 & 5.7 & 3.7 & 1764 \\
\hline Madras (3) & I & 2.6 & 6.7 & 1.6 & 4.7 & 2.0 & 3044 \\
\hline New Delhi & I & 7.1 & 9.1 & 7.5 & 7.8 & 6.2 & 2818 \\
\hline Neyveli & I & 4.3 & 10.3 & 3.3 & 4.8 & 3.3 & 3273 \\
\hline Orissa & I & 2.3 & 3.3 & 1.0 & 2.6 & 2.2 & 1240 \\
\hline
\end{tabular}


Table 3. - continued.

\begin{tabular}{|c|c|c|c|c|c|c|c|}
\hline \multirow[t]{2}{*}{ Centre } & \multirow[t]{2}{*}{ Video } & \multicolumn{5}{|c|}{ Prevalence in the last year } & \multirow[t]{2}{*}{$\mathrm{n}$} \\
\hline & & Wheeze & $\begin{array}{l}\text { Exercise } \\
\text { wheeze }\end{array}$ & $\begin{array}{c}\text { Night } \\
\text { wheeze }\end{array}$ & $\begin{array}{l}\text { Night } \\
\text { cough }\end{array}$ & $\begin{array}{c}\text { Severe } \\
\text { wheeze }\end{array}$ & \\
\hline Pune & I & 0.7 & 1.4 & 0.6 & 0.9 & 0.9 & 2579 \\
\hline Regional total & & 2.9 & 5.5 & 2.3 & 3.7 & 2.5 & 30043 \\
\hline $\begin{array}{l}\text { Western Europe } \\
\text { Austria }\end{array}$ & & & & & & & \\
\hline $\begin{array}{l}\text { Austria } \\
\text { Salzburo }\end{array}$ & & 5.5 & 15.0 & 3.3 & 8.8 & 3.1 & 4876 \\
\hline Salzburg & E & 5.0 & 14.7 & 3.1 & 9.1 & 3.2 & 3370 \\
\hline Urfahr-Ümgebung & $\mathrm{E}$ & 6.5 & 15.6 & 3.7 & 8.2 & 2.9 & 1506 \\
\hline France & & 8.3 & 22.8 & 4.4 & 14.9 & 4.6 & 8802 \\
\hline Marseilles & E & 9.1 & 23.1 & 5.1 & 15.2 & 5.5 & 3409 \\
\hline Strasbourg & $\mathrm{E}$ & 7.9 & 22.5 & 3.9 & 14.8 & 4.1 & 5393 \\
\hline Germany & & 5.3 & 16.7 & 3.6 & 10.2 & 3.6 & 7019 \\
\hline Greifswald & E & 4.8 & 17.2 & 3.5 & 9.7 & 3.0 & 3020 \\
\hline Münster & $\mathrm{E}$ & 5.6 & 16.4 & 3.8 & 10.6 & 4.1 & 3999 \\
\hline Italy & & 5.3 & 16.9 & 2.1 & 13.2 & 2.8 & 18181 \\
\hline Ascoli Piceno & I & 6.0 & 19.8 & 1.7 & 9.6 & 2.3 & 1089 \\
\hline Cremona & I & 4.1 & 13.1 & 1.4 & 12.0 & 2.3 & 1191 \\
\hline Emilia-Romagna & I & 5.8 & 17.5 & 1.9 & 12.4 & 3.0 & 3778 \\
\hline Empoli & I & 5.0 & 17.7 & 1.5 & 17.0 & 3.2 & 1038 \\
\hline Firenze & I & 3.9 & 17.8 & 1.5 & 14.0 & 2.3 & 1167 \\
\hline Milano & I & 5.5 & 19.1 & 1.9 & 16.8 & 3.7 & 3357 \\
\hline Roma & I & 4.8 & 15.9 & 1.6 & 11.8 & 2.8 & 3147 \\
\hline Torino & I & 5.3 & 15.7 & 1.5 & 14.7 & 2.3 & 1239 \\
\hline Verona & I & 5.8 & 14.4 & 4.8 & 10.6 & 2.1 & 2175 \\
\hline \multicolumn{8}{|l|}{ Portugal } \\
\hline Funchal & E & 6.2 & 14.4 & 3.3 & 10.2 & 5.0 & 3407 \\
\hline Spain & & 7.8 & 25.7 & 4.8 & 16.5 & 3.9 & 24842 \\
\hline Barcelona & I & 7.4 & 21.9 & 4.7 & 16.3 & 4.1 & 2984 \\
\hline Bilbao & $\mathrm{E}$ & 9.8 & 27.6 & 6.1 & 16.7 & 5.3 & 3167 \\
\hline Cartagena & $\mathrm{E}$ & 7.7 & 22.9 & 4.1 & 12.8 & 3.7 & 3013 \\
\hline Castellón & $\mathrm{E}$ & 5.8 & 18.9 & 3.9 & 18.7 & 2.6 & 3089 \\
\hline Cádiz & $\mathrm{E}$ & 8.9 & 24.5 & 6.4 & 16.4 & 4.3 & 3238 \\
\hline Pamplona & $\mathrm{E}$ & 6.9 & 24.6 & 3.8 & 15.8 & 3.4 & 3040 \\
\hline Valencia & $\mathrm{E}$ & 9.6 & 35.4 & 5.1 & 17.9 & 4.3 & 3140 \\
\hline Valladolid & $\mathrm{E}$ & 6.3 & 29.0 & 3.9 & 17.1 & 3.2 & 3171 \\
\hline Regional total & & 6.7 & 20.6 & 3.7 & 13.9 & 3.7 & 67127 \\
\hline Global total & & 7.3 & 13.7 & 3.7 & 11.2 & 4.5 & 304796 \\
\hline
\end{tabular}

Data are presented as percentages. E: European version of video; I: International version of video. *: data recoded to eliminate inconsistent responses.

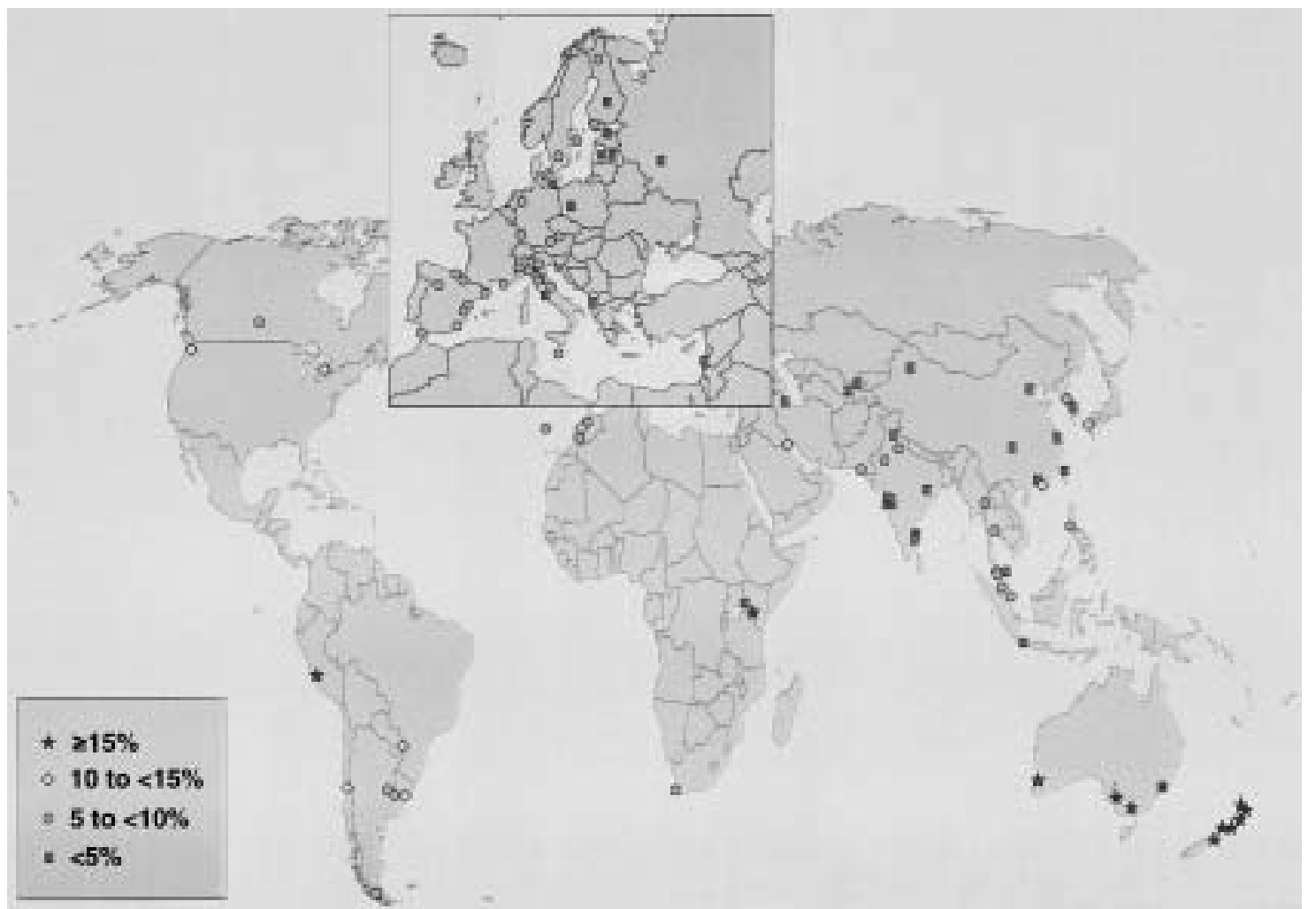

Fig. 5. - World map for the 13-14 yr old age group, for the video scene showing a person wheezing while at rest. The percentage of children who answered "yes" to the question "Has your breathing ever been like this... in the last year?" is shown for each centre, with distinct colour symbols used for different prevalence ranges. 


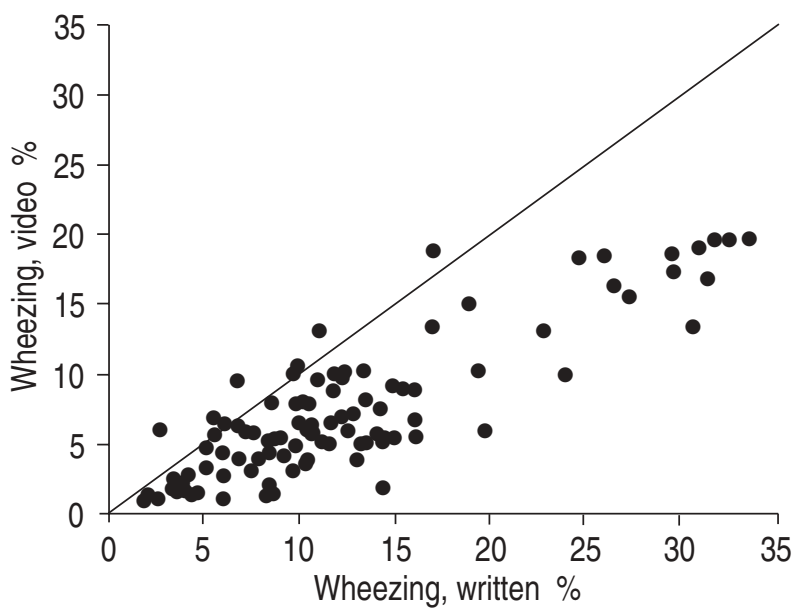

Fig. 6. - Scatter plots for the percentage of the 13-14 yr old age group responding "yes" for wheezing in the last year (video questionnaire) against "wheezing or whistling in the chest in the last 12 months". The xaxis shows wheezing for the written questionnaire and the y-axis wheezing for the video questionnaire. The line of identity is shown.

at rest in the last year, common to both video versions; $33 \%$ of these centres used the European version while $67 \%$ used the International version. There was a wide range of prevalence rates between countries (1.0-18.5\%), showing more than 15 -fold differences. The lowest prevalences $(<3 \%)$ were found in Albania, China, Estonia, India, Indonesia, Latvia, Russia and Uzbekistan and the highest prevalences $(>15 \%)$ in Australia, New Zealand and Peru. The between-country variation was greater than the within-country variation for all questions in the last 12 months (1.8-2.5 times larger), except for night cough (1.0).

Wheezing with exercise in the last year was more common than wheezing in the last year in all countries except for Pakistan and Singapore. Night cough in the last year was more common than wheezing in the last year in all countries except for Japan, Kuwait, Pakistan, Peru, Russia, Singapore and Taiwan.

The overall pattern of international differences shown by the 13-14 yr olds who took part in both the video and written questionnaires was similar (figs. 1 and 5), with a correlation of $\mathrm{r}=0.76(\mathrm{p}<0.0001)$ (fig. 6). Video prevalence estimates were generally lower than written estimates for comparable questions in centres in the majority (Š75\%) of countries, except that "severe asthma" on the video questionnaire usually exceeded "severe wheezing limiting speech" on the written questionnaire. Some of the greatest differences (>fivefold) between the video and written questionnaires for wheezing in the last year were found in Latvia and Uzbekistan. Large absolute differences $(>13 \%)$ were found in centres in Australia, Canada and Finland.

\section{Discussion}

The ISAAC study has demonstrated, by means of simple standardized questionnaires, that there are large variations in the prevalence of asthma symptoms throughout the world. The self-reported 12 month prevalence of wheezing among 13-14 yr olds between countries ranged from $2.1 \%$ in Indonesia to $32.2 \%$ in the UK. Parental reported 12 month prevalence of wheezing in 6-7 yr olds ranged from $4.1 \%$ in Indonesia to $32.1 \%$ in Costa Rica. The highest values for 12 month prevalence of wheeze were found in developed English-speaking countries, and some Latin American non-English-speaking countries (e.g. Peru and Costa Rica). There were considerable variations within regions, e.g. the 12 month prevalence in the 13-14 yr old age group varied within Europe from $<5 \%$ in centres in Albania, Georgia, Greece, Italy, Romania and Russia to $>30 \%$ in the UK, and within Latin America from $<10 \%$ in centres in Argentina, Chile and Mexico to $>25 \%$ in centres in Brazil and Peru.

The analysis shows that there is consistently more variation between countries than within countries. Three countries with a very large number of centres were represented across the range of prevalences, India with 14 centres representing the low prevalence group, Italy with 14 centres representing the middle prevalence group and the UK with 15 centres representing the high prevalence group. However, it must be remembered that the countries, and centres within countries were self selected, and it is possible that countries with larger within-country variation did not participate.

Although previous international comparisons [1, 1317] have drawn attention to variations in the prevalence of childhood asthma, the ISAAC project comprises by far the most extensive international survey of asthma symptoms ever performed. Adherence to the protocol ensured that, within centres, there was standardization of the questionnaires and methods of administration and that schools were selected without bias from the defined geographical area. These centres, however, may not represent the prevalence of asthma symptoms throughout the whole country and no comparison could be made between rural and urban areas. Moreover, there may have been important variation within some centres, for example between affluent and poor areas, which the ISAAC Phase One study did not examine. Targets for the sample size ensured there was sufficient precision in the prevalence estimates obtained. By recommending that at least half of the survey be conducted before the main pollen season it was hoped that variation due to seasonal factors would be reduced; in the event, studies in New Zealand [18], the UK and Germany have found that the 12 month period prevalence of the various wheezing symptoms is not significantly affected by the season of the survey.

The only other comparable international survey of asthma is the European Community Respiratory Health Survey (ECHRS) [19, 20], which studied males and females aged 20-44 yrs, mainly from European centres. Among the 13 centres in 10 countries that were reported in both studies, the ranking of prevalence of wheeze in the last 12 months was similar, with the English-speaking countries (Australia, New Zealand, Republic of Ireland and the UK) having the highest and Italy and Greece the lowest rates.

There is, as yet, no accepted definition of asthma and the identification of asthma by questionnaire remains a contentious issue. The ISAAC collaboration agreed that the cardinal symptom of asthma would be that which reflects variable narrowing of the airways and that this is best described (in English) as "wheezing or whistling in the chest". The questionnaire incorporated questions taken 
from pre-existing questionnaires with sensitive (although not necessarily specific) questions for asthma and more specific questions which related to the severity of asthma. In recent years a number of studies have compared responses to the ISAAC core wheezing questions with other indicators of asthma, including physician diagnosis [21], other questionnaires [22] and physiological measures [9, $10,22,23]$. These indicate a level of sensitivity and specificity which is acceptable for the purposes of multicentre international comparisons.

While the significance of very mild wheezing may be questioned, the occurrence of frequent, sleep-disturbing or speech-limiting attacks is accepted widely as clinically important. There were large international variations in the prevalence of these three more specific symptoms and, broadly, the worldwide pattern of prevalence of more severe symptoms followed that of any 12 month wheezing. However, the order of countries ranking highest for the prevalence of "sleep disturbance due to wheezing one or more nights per week in the last 12 months" and "wheeze severe enough to limit speech to only one or two words at a time between breaths" was different from 12 month wheeze, while four or more attacks in the last 12 months was similar to 12 month wheeze. The explanations for the variation between prevalence and severity would be of particular interest and may include differences in the management of asthma between countries, although information on asthma management was not collected in ISAAC Phase One.

The proportion of wheezy children with severe asthma symptoms varied little with the increasing prevalence of wheezy children in the population. This means the high prevalences found in some centres are not explained by excessive inclusion of children with mild wheeze. These findings suggest that factors affecting the prevalence of asthma also affect the severity of asthma, and the factors which especially affect the prevalence of severe asthma should be examined.

The validity of the questionnaire is likely to have varied across cultures and languages. Some languages do not have an equivalent of "wheezing" as understood by English speakers. The high prevalence in English-speaking countries, especially in those most accustomed to using "wheezing" as a term in surveys, suggests there may be some language bias associated with the written questionnaire, as suggested by the findings of the ECHRS [20], or that the population has a higher awareness of asthma. However, high prevalences were also observed in Spanish- and Portuguese-speaking countries in South America, suggesting that other factors are also likely to be important in determining the prevalence of asthma symptoms. There were also large variations in prevalence within Spanish, Portuguese and Chinese languages. The overall pattern of international differences observed with the written questionnaire was maintained among those centres that employed the video questionnaire. Positive responses to the video were generally lower than for similar questions with the written questionnaire. A possible reason is that signs that are obvious enough to be visible or audible on a video are likely to represent more severe symptoms than the full spectrum from mild to severe asthma covered by the written questionnaire. It would be helpful to obtain further validation studies in centres of low prevalence.
There was also considerable variation in the prevalence of a positive response to the question concerning whether the child had ever had "asthma". This question probably indicates the level of perceived or diagnosed asthma in the various centres but is a less reliable measure for epidemiological purposes [24]. Even in those countries with the lowest prevalences (Albania, Estonia, Indonesia, Latvia and Uzbekistan 1.4-1.7\%) asthma is clearly an important medical diagnosis. Over $20 \%$ of children in the countries of highest prevalence (Australia, Costa Rica, New Zealand, Oman, Peru, Singapore and the UK) reported "asthma".

The prevalence of exercise-related wheeze in the last 12 months differed from 12 month wheeze in both age groups, being consistently higher in older children and lower in younger children. This may be real or represent under-reporting by parents and over-reporting by adolescents, or different interpretation of wheezing questions. These differences indicate the need for further examination of the validity of these questions. Higher rates of reporting of symptoms by adolescents compared with their parents has been noted in other studies ([25], Strachan personal communication), and rates of symptoms in adolescents are generally similar to those in young adults, suggesting that parental reporting may underestimate true symptoms. In contrast, the prevalence of night cough was consistent in both age groups, and showed a higher prevalence than any other question. Although there was a very strong correlation with wheezing in the last 12 months, the higher prevalence of night cough suggests that this question, in addition to estimating asthma, may be measuring other respiratory conditions. Parents may have a higher awareness of night cough because it disturbs their sleep.

The prevalence of asthma symptoms was greater in males in the younger age group. There was a mixed picture in the older age group, with considerable variation between countries but, on average, females had slightly higher prevalence than males. It is possible that the emergence of a female predominance of asthma in older adolescents and adults may depend on physical maturation, which is likely to occur at different ages in the range of countries studied. This pattern of sex differences has been reported in other studies in Western countries [26], including one large study which used ISAAC questions systematically across the age range 5-17 yrs [27]. It therefore appears unlikely that the present findings with respect to sex differences can be attributed entirely to bias arising from the different methods of questionnaire administration in the younger and older age groups.

A striking feature of these data is the high rates of asthma symptoms in countries whose predominant language is English and further study of possible aetiological factors common to these countries is a high priority. In contrast, while high rates of asthma symptoms were also found in some non-English-speaking countries, rates were not uniformly high among countries sharing the same language. For example, Peru and Costa Rica had a much higher prevalence than Spain, Brazil had a higher prevalence than Portugal and Hong Kong had a higher prevalence than China. The variations are unlikely to be explained entirely by interpretation of language, given the similar trends found between written and video questionnaires, and provide important opportunities for further study. 
This large international collaboration, examining the prevalence of reported asthma symptoms in children, has set the framework from which to study a variety of factors contributing to asthma and provides a baseline from which to measure future trends. The most important outcome of ISAAC will be to advance the understanding of the causes of asthma, by studying factors acting at a population level. Although genetic factors are important risk factors for individuals within populations, migrant studies indicate they are unlikely to be responsible for the large variations in asthma which exist between populations and cannot be responsible for the recent upward trends within populations [28]. Environmental factors are likely to be more important and offer the greatest opportunities for prevention. An attractive current theory, supported by experimental evidence, is that increased hygiene and healthcare in Western countries has altered the pattern of exposure to infection in early life in such a way as to predispose the immune system towards the atopic response [29, 30]. There are also a number of theories concerning the provocation of asthma by various agents including aeroallerg-ens, diet, indoor and outdoor pollution. The ISAAC group is now using the prevalence data reported here to conduct ecological studies between populations to test these and other hypotheses.

ISAAC Phase Two studies are now planned or underway in a number of countries and regions. Measures of bronchial hyperresponsiveness are being used to determine whether these support the questionnaire results. Measures of atopy (using allergen skin tests and immunoglobulin $\mathrm{E}$ measurements) are being used to determine the extent to which variations in wheezing illness are reflected in variations in atopy. Phase Two studies will also systematically obtain environmental data such as indoor exposure to allergens and pollutants to contribute to the ecological analyses.

This worldwide study of asthma symptoms is a major step forward in using population differences in prevalence to gain new insights into the causes of asthma. The simplicity of the questionnaire method enabled a wide range of countries to participate but was necessarily accompanied by some uncertainty as to international comparability. However, the large variations found are most unlikely to be explained by methodological factors alone. The existence of such wide variation provides an epidemiological opportunity for new investigations into the causes of asthma.

\section{Appendix: Centres and principal investigators}

\begin{tabular}{|c|c|}
\hline \multicolumn{2}{|l|}{ Albania } \\
\hline Tiranë & $\begin{array}{l}\text { A. Priftanji, Allergy Clinic, } \\
\text { University Hospital }\end{array}$ \\
\hline \multicolumn{2}{|l|}{ Algeria } \\
\hline Algiers & $\begin{array}{l}\text { A. Bezzaoucha, Chu Bab el } \\
\text { Oued, Service d'Epidemiologie }\end{array}$ \\
\hline \multicolumn{2}{|l|}{ Argentina } \\
\hline Buenos Aires, Rosario & $\begin{array}{l}\text { N. Salmun*, Fundación para el } \\
\text { Estudio del Asma y otras } \\
\text { Enfermedades Alérgicas }\end{array}$ \\
\hline \multicolumn{2}{|r|}{ 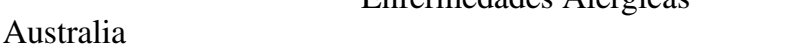 } \\
\hline Adelaide & $\begin{array}{l}\text { D. Kennedy, Respiratory Medic- } \\
\text { ine, Adelaide Children's Hospital }\end{array}$ \\
\hline Melbourne & $\begin{array}{l}\text { C. Robertson*, Dept of } \\
\text { Thoracic Medicine, Royal } \\
\text { Children's Hospital }\end{array}$ \\
\hline Perth & $\begin{array}{l}\text { L. Landau, Faculty of Medicine } \\
\text { and Dentistry, University of } \\
\text { Western Australia }\end{array}$ \\
\hline Sydney (6-7 yr) & $\begin{array}{l}\text { J. Peat, Dept of Medicine, } \\
\text { University of Sydney }\end{array}$ \\
\hline Sydney (13-14 yr) & $\begin{array}{l}\text { A. Bauman, School of } \\
\text { Community Medicine, } \\
\text { University of New South Wales }\end{array}$ \\
\hline \multicolumn{2}{|r|}{ 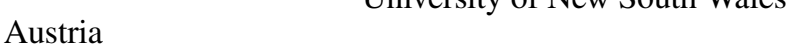 } \\
\hline Salzburg & J. Riedler*, Kinderspital \\
\hline Urfahr-Umgebung & $\begin{array}{l}\text { G. Haidinger, Dept of } \\
\text { Epidemiology, Institute of } \\
\text { Tumorbiology/Cancer } \\
\text { Research University of Vienna }\end{array}$ \\
\hline \multicolumn{2}{|l|}{ Belgium } \\
\hline Antwerp & $\begin{array}{l}\text { P.A. Vermeire, Dept of } \\
\text { Respiratory Medicine, } \\
\text { University of Antwerp }\end{array}$ \\
\hline \multicolumn{2}{|l|}{ Brazil } \\
\hline Curitiba & $\begin{array}{l}\text { N.A. Rosário, Dept of Pediatrics } \\
\text { Federal University of Parana }\end{array}$ \\
\hline Porto Alegre & $\begin{array}{l}\text { R. Stein, Respiratory Sciences } \\
\text { Center, College of Medicine }\end{array}$ \\
\hline
\end{tabular}

Albania

Algeria

Algiers

gentina

Buenos Aires, Rosario

Estudio del Asma y otras ine, Adelaide Children's Hospital C. Robertson*, Dept of Children's Hospital and Dentistry, University of

University of Sydney

Community Medicine,

J. Riedler*, Kinderspital

G. Haidinger, Dept of

Tumorbiology/Cancer

P.A. Vermeire, Dept of

Respiratory Medicine,

N.A. Rosário, Dept of Pediatrics

R. Stein, Respiratory Sciences

Center, College of Medicine
Recife

Salvador

São Paulo

Canada

Hamilton

Saskatoon

Chile

Central Santiago

Punta Arenas

South Santiago

Valdivia

China

Beijing

Chongqing

Guangzhou

Shanghai

Wulumuqi
P.G.dM. Bezerra, Instituto Matterno Infantil de Pernambuco L.S.dF. Souza, Faculty of Medicine, Federal University of Bahia D. Solé*, Division of Allergy, Universidade Federal de São Paulo

M. Sears*, Firestone Regional Chest \& Allergy Unit, St. Joseph's Hospital, McMaster University B. Taylor, IWK - Grace Emergency

I. Sanchez, Departamento de Pediatria, Universidad Catolica de Chile L. Amarales, Children's Respiratory Service, Regional Hospital "Lautaro Navarro" E. Cortez, Dept of Paediatric Respiratory Medicine, Hospital El Pino, University of Santiago de Chile M. Calvo-Gil, Instituto de Pediatria, Facultad de Medicina, Universidad Austral de Chile

Y. Chen*, Capital Institute of Pediatrics

K-H. Chen, Children's Hospital N-S. Zhong, Director, Guangzhou Institute of Respiratory Disease M. Bao-Shan, Central Hospital of Zhabei District of Shanghai M-L. Xiao, Dept of Paediatrics First Affiliated Hospital, Xinjiang Medical College 
Costa Rica

Costa Rica

Estonia

Narva, Tallinn

Ethiopia

Addis Ababa

Jima

Finland

Helsinki

Kuopio County

Turku and Pori County

France

Marseille

Montpellier

Pessac

Strasbourg

West Marne

Georgia

Kutaisi

Tbilisi

Germany

Greifswald

Münster

Greece

Athens

Hong Kong

Hong Kong (6-7 yr) Y.L. Lau, Dept of Paediatrics,

Hong Kong (13-14 yr) C. Lai*, Dept of Medicine, ChiIndia

Akola University of Hong Kong nese University of Hong Kong

M.E. Soto-Quirós, Unidad de Enseñanza, Hospital Nacional de Niños, San José

M-A. Riikjärv*, Tallinn

Children's Hospital

K. Melaku*, Dept of Internal

Medicine, Addis Ababa

University

B. Seyoum, Dept of Internal

Medicine, Addis Ababa

University

M. Kajosaari, Helsinki

University Central Hospital

J. Pekkanen*, Dept of

Environmental Epidemiology,

National Public Health

Institute

L. Soininen, Provincial

Administrative Board of

Lapland

University Central Hospital

D. Charpin*, Service de

Pneumologie-Allergologie,

P. Godard, Clinic des

Maladies Respiratoires,

Hôpital Arnaud de Villeneuve

A. Taytard, Centre Hospitalier

Universitaire de Bordeaux,

Service des Maladies

Respiratoires

G. Pauli, Service de

Pneumologie, Hôpital Civil

I. Annesi, INSERM, U. 169:

Recherches en Epidémiologie, Villejuif Cedex

N. Khetsuriani, USA Center

for Disease Control and

Prevention Project in Georgia

A. Gamkrelidze*, Department of Allergology, Tbilisi

Medical University

A. Kramer, Institut für

Hygiene und Umweltmedizin

U. Keil*, Institut für Epidemiologie und Sozialmedizin, Westfälische Wilhelms Universität

C. Gratziou, Pulmonary and Critical Care Dept, Evgenidio Hospital

R. Maheshwari, Bhartia Municipal Hospital
Hôpital Nord
Bombay (16)

Bombay (17)

Bombay (18)

Borivali

Chandigarh

Jodhpur

Kottayam

Madras (2)

Madras (3)

New Delhi

Neyveli

Orissa

Pune

Indonesia

Bandung

Iran

Rasht, Tehran

Italy

Âscoli Piceno

Cosenza

Cremona

Emilia-Romagna

Empoli

Firenze

Frosinone

Milano

Roma

Siena
M.K. Joshi, K.B. Bhabha

Hospital

U.A. Pai, Pediatrician

K. Raghavan, L.T.M. Medical

College

V.A. Khatav, Dr Khatav's

Mother and Child Hospital

L. Kumar, Postgraduate

Institute of Medical Education and Research

K.C. Jain, Pioneer Medical

Centre

T.U. Sukumaran, Institute of Child Health Medical College

S. Rajajee, Childs Trust

Hospital

N. Somu, Institute of Child

Health

K. Chopra, Maulana Azad

Medical College

G. Jayaraj, Occupational

Health, Neyveli Lignite

Corporation Hospital

P.K. Kar, Ispat Hospital

N.M. Hanumante, Consulting

Pediatrician, "Happy Clinic"

B. Karnen*, Dept of

Medicine, Medical Faculty,

University of Indonesia

M-R Masjedi*, National

Research Institute of

Tuberculosis and Lung Disease,

Shaheed Beheshti University

of Medical Sciences and

Health Services

S. Bonini, Consultorio

Familiare USL 24

E. Bonci, Università degli

Studi di Roma "La Sapienza",

Istituto di Clinica Pediatrica

F. Rusconi, Isituto di Clinica di

Perfezionamento, Azienda

Ospedaliera, Clinica Pediatrica

"G. e D. De Marchi"

M. Biocca, CDS Aziende USL,

Città di Bologna e Ravenna

L. Chetoni, Centro Studi

Prevenzione Oncologica

(CSPO), U.O. Epidemiologia

USL 10/E

E. Chellini, Centro Studi Prevenzione Oncologica (CSPO), U.O. Epidemiologia USL 10/E

R. Ronchetti, Università degli

Studi di Roma "La Sapienza",

Istituto di Clinica Pediatrica,

Policlinico Umberto $1^{\circ}$

L. Bisanti, Regione Lombardia

Servizio di Epidemiologia

F. Forastiere*, Osservatorio

Epidemiologico

E. Renzoni, Istituto Malattie

Respiratorie, Università di

Siena, Ospedale Le Scotte 


\begin{tabular}{|c|c|c|c|}
\hline Torino & $\begin{array}{l}\text { G. Ciccone, Servizio } \\
\text { Universiario, Epidemiologia } \\
\text { dei Tumori, Azienda } \\
\text { Ospedaliera S.G. Battista }\end{array}$ & $\begin{array}{l}\text { Nelson } \\
\text { Wellington }\end{array}$ & $\begin{array}{l}\text { R. Mackay, Dept of Paediatrics, } \\
\text { Nelson Hospital } \\
\text { J. Crane, Dept of Medicine, } \\
\text { Wellington School of Medicine }\end{array}$ \\
\hline Trento & $\begin{array}{l}\text { S. Piffer, Azienda Provinciale } \\
\text { Sanitaria, Osservatorio } \\
\text { Epidemiologico }\end{array}$ & $\begin{array}{l}\text { Nigeria } \\
\text { Ibadan }\end{array}$ & $\begin{array}{l}\text { B.O. Onadeko, Dept of Medic- } \\
\text { ine, University College Hospital }\end{array}$ \\
\hline $\begin{array}{l}\text { Verona } \\
\text { Viterbo }\end{array}$ & $\begin{array}{l}\text { A. Boner, Clinica Pediatrica, } \\
\text { Università di Verona } \\
\text { G. Corbo, Servizio di Fisio- } \\
\text { patologia Respiratoria, Univer- } \\
\text { sità Cattolica del Sacro Cuore }\end{array}$ & $\begin{array}{l}\text { Oman } \\
\text { Al-Khod }\end{array}$ & $\begin{array}{l}\text { B.M.S. Al Riyami, Dept of } \\
\text { Medicine, Sultan Qaboos } \\
\text { University }\end{array}$ \\
\hline $\begin{array}{l}\text { Japan } \\
\text { Fukuoka }\end{array}$ & $\begin{array}{l}\text { S. Nishima, National Minami } \\
\text { Fukuoka Chest Hospital }\end{array}$ & Karachi & $\begin{array}{l}\text { Z.A. Bhutta, Faculty of Health } \\
\text { Sciences, The Aga Khan } \\
\text { University }\end{array}$ \\
\hline $\begin{array}{l}\text { Kenya } \\
\text { Eldoret }\end{array}$ & $\begin{array}{l}\text { F.O. Esamai, Dept of Child } \\
\text { Health and Paediatrics, Moi } \\
\text { University } \\
\text { J.A. Odhiambo*, Respiratory } \\
\text { Diseases Research Unit, Kenya } \\
\text { Medical Research Institute }\end{array}$ & $\begin{array}{l}\text { Panama } \\
\text { David-Panamá } \\
\text { Paraguay } \\
\text { Asunción }\end{array}$ & $\begin{array}{l}\text { J.A. Guggiari-Chase, Sociedad } \\
\text { Paraguaya de Alergia e } \\
\text { Immunologia }\end{array}$ \\
\hline $\begin{array}{l}\text { Kuwait } \\
\text { Kuwait } \\
\text { Latvia }\end{array}$ & $\begin{array}{l}\text { J.A. Al-Momen, Al-Amiri } \\
\text { Hospital }\end{array}$ & eru & $\begin{array}{l}\text { P. Chiarella, Departamento de } \\
\text { Pediatria, Universidad } \\
\text { Peruana Cayetano Heredia }\end{array}$ \\
\hline Riga, Rural Latvia & $\begin{array}{l}\text { M. Leja*, Latvia Medical Aca- } \\
\text { emy, Human Ecology Institute }\end{array}$ & $\begin{array}{l}\text { Philippines } \\
\text { Metro Manilla }\end{array}$ & $\begin{array}{l}\text { F. Cua-Lim, St Lukes Medical } \\
\text { Center }\end{array}$ \\
\hline Beirut & $\begin{array}{l}\text { F.M. Ramadan, Dept of } \\
\text { Medicine, American } \\
\text { University of Beirut }\end{array}$ & $\begin{array}{l}\text { Poland } \\
\text { Krakow }\end{array}$ & $\begin{array}{l}\text { G. Lis*, 1st Dept of Pediatrics, } \\
\text { Polish-American Children's } \\
\text { Hospital }\end{array}$ \\
\hline Alor Setar & $\begin{array}{l}\text { K.H. Teh, Dept of Paediatrics, } \\
\text { Hospital Alor Setar } \\
\text { W.Y. Lim, Dept of Paediatrics, } \\
\text { Ipoh Hospital }\end{array}$ & Poznan & $\begin{array}{l}\text { A. Breborowicz, Dept of Pedi- } \\
\text { atric Pulmonology, University } \\
\text { of Medical Sciences }\end{array}$ \\
\hline $\begin{array}{l}\text { Klang Valley } \\
\text { Kota Bharu }\end{array}$ & $\begin{array}{l}\text { J. de Bruyne*, Dept of Paedia- } \\
\text { trics, University Hospital } \\
\text { B.S. Quah, School of Medical }\end{array}$ & Funchal & $\begin{array}{l}\text { F. Drummond Borges, Serviço } \\
\text { de Medicina, Centro Hosp- } \\
\text { italar do Funchal }\end{array}$ \\
\hline 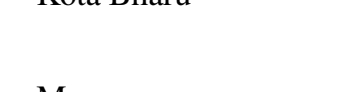 & $\begin{array}{l}\text { Sciences, University Sains } \\
\text { Malaysia }\end{array}$ & Lisbon & $\begin{array}{l}\text { J. Rosado Pinto*, Serviço de } \\
\text { Immunoalergologia, Hospital }\end{array}$ \\
\hline Muar & $\begin{array}{l}\text { K.W. Chum, Johor Child Spe- } \\
\text { cialist Clinic, Hospital Muar }\end{array}$ & Portimão & $\begin{array}{l}\text { de Dona Estefânia } \\
\text { C. Nunes, Administração } \\
\text { Regional de Saúde do Algarve }\end{array}$ \\
\hline Malta & $\begin{array}{l}\text { S. Montefort, Dept of } \\
\text { Medicine, University of Malta } \\
\text { Medical School }\end{array}$ & Porto & $\begin{array}{l}\text { (ARS) } \\
\text { J. Lopes dos Santos, Serviço } \\
\text { de Pediatria, Hospital de }\end{array}$ \\
\hline $\begin{array}{l}\text { Mexico } \\
\text { Cuernavaca }\end{array}$ & & & Matosinhos \\
\hline Cuernavaca & $\begin{array}{l}\text { Pomieu, Centro } \\
\text { Panamericano de Ecologia } \\
\text { Humana y Salud }\end{array}$ & $\begin{array}{l}\text { Republic of Ireland } \\
\text { Romania }\end{array}$ & $\begin{array}{l}\text { L. Clancy; Respiratory Research } \\
\text { Centre, St James's Hospital }\end{array}$ \\
\hline Casablanca, Marrakech & $\begin{array}{l}\text { Z. Bouayad*, Service de } \\
\text { Pneumologie, Centre Hospi- } \\
\text { talier Ibnou Rochd }\end{array}$ & Cluj & $\begin{array}{l}\text { D. Dumitrascu, Allergy Dept, } \\
\text { University Medicine and } \\
\text { Pharmacology }\end{array}$ \\
\hline $\begin{array}{c}\text { Rabat } \\
\text { New Zealand }\end{array}$ & $\begin{array}{l}\text { A. Bennis, Résidence du } \\
\text { Minaret }\end{array}$ & $\begin{array}{l}\text { Russia } \\
\text { Moscow }\end{array}$ & $\begin{array}{l}\text { R. Khaitov, Institute of } \\
\text { Immunology, National }\end{array}$ \\
\hline Auckland & $\begin{array}{l}\text { M.I. Asher*, Dept of Paediat- } \\
\text { rics, University of Auckland }\end{array}$ & Singapore & Research Center \\
\hline Bay of Plenty & $\begin{array}{l}\text { C. Moyes, Dept of Child } \\
\text { Health, Whakatane Hospital }\end{array}$ & Singapore & $\begin{array}{l}\text { B-W. Lee, Children's Medical } \\
\text { Center, National University }\end{array}$ \\
\hline Christchurch & $\begin{array}{l}\text { P. Pattemore, Dept of } \\
\text { Paediatrics, Christchurch }\end{array}$ & South Africa & Hospital \\
\hline Hawke's Bay & $\begin{array}{l}\text { School of Medicine } \\
\text { D. Barry, Dept of Paediatrics, } \\
\text { Memorial Hospital }\end{array}$ & Cape Town & $\begin{array}{l}\text { H. Nelson, Dept of } \\
\text { Community Health Medical } \\
\text { School, Observatory } 7925\end{array}$ \\
\hline
\end{tabular}


South Korea

Provincial Korea, Seoul S-I. Lee*, Samsung Medical Center, Dept of Pediatrics

Spain

Barcelona

R.M. Busquets, Servicio de Pediatría, Hospital del Mar, Universidad Autónoma de Barcelona

Bilbao

A.D. Rubio, Dept of Pediatrics Basque Country University

Cádiz

A.R. Asensio, Sevicio

Andaluz de Salud, Districto

Bahia-Vejer

Cartagena L. García-Marcos*

Universidad de Murcia,

Dirección de Salud Area II

Castellón

A. Arnedo-Pena, Sección de

Epidemiologia, Dirección

Territorial Sanidad

Pamplona

F. Guillén Grima, Universidad

Pública de Navarra, Dept de

Ciencias de la Salud

Valencia M.M.M. Suárez-Varela, Dept de Medicina Preventiva i Salut Pública, Bromatologia,

Toxicologia i Medicina Legal

Valladolid

A. Blanco Quirós, Dept

Pediatria, Facultad de

Medicina

Sweden

Linköping

Stockholm/Uppsala

N-I. Kjellman, Dept of

Pediatrics, University Hospital

T. Foucard, Dept of

Paediatrics, Uppsala

University Children's Hospital

Taiwan

Taipei

Thailand

Bangkok
K-H. Hsieh*, Director, Chang Gung Children's Hospital

P. Vichyanond*, Dept of

Pediatrics, Faculty of

Medicine, Mahidol University
Chiang Mai

United Kingdom

Anglia and Oxford, North East and Yorkshire, North Thames, North West, South and West, South Thames, Scotland, Trent, Wales, West Midlands

H.R. Anderson*, Dept of

Public Health Sciences,

St George's Hospital

Medical School

Guernsey

Isle of Man

Jersey

Sunderland

Surrey/Sussex

Uruguay

Montevideo

USA

Chicago

Seattle

Uzbekistan

Samarkand, Tashkent T. Aripova*, Institute of Immunology, Uzbek Ministry of Public Health
Writing Group: M.I. Asher, University of Auckland, Auckland, New Zealand; H.R. Anderson, St George's Hospital Medical School, London, UK; A.W. Stewart, Univeristy of Auckland, Auckland, New Zealand; J. Crane, Wellington School of Medicine, Wellington, New Zealand.

ISAAC Steering Committee: N. Aitt-Khaled (Regional Coordinator), IUATLD, Paris, France; G. Anabwani (Regional Coordinator), Princess Marina Hospital, Gaborone, Botswana; H.R. Anderson, St George's Hospital Medical School, London, UK; M.I. Asher (Chairperson of the ISAAC Steering Committee and ISAAC Executive, Director of the ISAAC International Data Centre, Regional Coordinator), University of Auckland, Auckland, New Zealand; R. Beasley (ISAAC Executive, Coordinator Phase One), Wellington School of Medicine, Wellington, New Zealand; B. Björkstén (ISAAC Executive, Regional Coordinator), University Hospital, Linköping, Sweden; M.L. Burr, University of Wales, College of Medicine, Cardiff, UK; T.O. Clayton (ISAAC International Data Centre), University of Auckland, Auckland, New Zealand; J. Crane, Wellington School of Medicine, Wellington, New Zealand; P. Ellwood (ISAAC International Data Cen- tre), University of Auckland, Auckland, New Zealand; U. Keil (Regional Coordinator), Universität Münster, Münster, Germany; C.K.W. Lai (Regional Coordinator), The Chinese University of Hong Kong, Hong Kong, China; J. Mallol (Regional Coordinator), University of Santiago, Santiago, Chile; F.D. Martinez (Regional Coordinator), University of Arizona, Tucson, USA; E.A. Mitchell (ISAAC International Data Centre), University of Auckland, Auckland, New Zealand; S. Montefort (Regional Coordinator), University of Malta Medical School, G'Mangia, Malta; N. Pearce, Wellington School of Medicine, Wellington, New Zealand; C.F. Robertson, Royal Children's Hospital, Parkville, Australia; J.R. Shah (Regional Coordinator), Jaslok Hospital and Research Centre, Mumbai, India; B. Sibbald, University of Manchester, Manchester, UK; A.W. Stewart (ISAAC International Data Centre), University of Auckland, Auckland, New Zealand; D.P. Strachan (ISAAC Executive), St George's Hospital Medical School, London, UK; E. von Mutius, Kinderklinik der Universität München, München, Germany; S.K. Weiland (ISAAC Executive, Coordinator Phase Two, Regional Coordinator), Universität Münster, Münster, Germany; H.C. Williams, University Hospital, Nottingham, UK. 


\begin{abstract}
Acknowledgements: The authors are indebted to the collaborators in the participating centres and all parents, children, teachers and other school staff who participated in the surveys. There are many field workers and funding agencies who supported data collection and national, regional and international meetings, including the meetings of the ISAAC Steering Committee. Unfortunately, these are too numerous to mention (they will be acknowledged elsewhere) but the authors particularly wish to thank the funders who supported the ISAAC International Data Centre including the Health Research Council of New Zealand, the Asthma and Respiratory Foundation of New Zealand, the National Child Health Research Foundation, the Hawke's Bay Medical Research Foundation, the Waikato Medical Research Foundation, Glaxo Wellcome New Zealand and Astra New Zealand, as well as Glaxo Wellcome International Medical Affairs for finding the regional co-ordinating centres.
\end{abstract}

\section{References}

1. Pearce N, Weiland SK, Keil U, et al. Self-reported prevalence of asthma symptoms in children in Australia, England, Germany and New Zealand: an international comparison using the ISAAC protocol. Eur Respir $J$ 1993; 6: 1455-1461.

2. Asher MI, Keil U, Anderson HR, et al. International study of asthma and allergies in childhood (ISAAC): rationale and methods. Eur Respir J 1995; 8: 483-491.

3. Strachan D, Sibbald B, Weiland SK, et al. Worldwide variations in prevalence of symptoms of allergic rhinoconjunctivitis in children: the International Study of Asthma and Allergies in Childhood (ISAAC). Pediatr Allergy Immunol 1997; 8: 161-176.

4. The International Study of Asthma and Allergies in Childhood (ISAAC) Steering Committee. Worldwide variation in the prevalence of symptoms of asthma, allergic rhinoconjunctivitis and atopic eczema: ISAAC. Lancet 1998; 351: 1225-1232.

5. Diem K, Lenter C, eds. Documenta Geigy Scientific Tables, 7th Edn. Basle, JR Geigy, 1970.

6. Burney PGJ, Laitinen LA, Perdrizet S, et al. Validity and repeatability of the IUATLD (1984) Bronchial Symptoms Questionnaire: an international comparison. Eur Respir J 1989; 2: 940-945.

7. Weiland SK, Kugler J, von Mutius E, et al. The language of pediatric asthma patients: verbal descriptors of symptoms in Germany. Monatsschr Kinderheilkd 1993; 141: 878-882 (in German).

8. Shaw RA, Crane J, Pearce N, et al. Comparison of a video questionnaire with the IUATLD written questionnaire for measuring asthma prevalence. Clin Exp Allergy 1992; 22: 561-568.

9. Shaw RA, Woodman K, Ayson M, et al. Measuring the prevalence of bronchial hyperresponsiveness in children. Int J Epidemiol 1995; 24: 597-602.

10. Lai CKW, Chan JKW, Wong G, et al. Comparison of the ISAAC video questionnaire (AVQ3.0) with the ISAAC written questionnaire for estimating asthma associated with bronchial hyperreactivity. Clin Exp Allergy 1997; 27: 540-545.

11. Rao JNK, Scott AJ. A simple method for the analysis of clustered binary data. Biometrics 1992; 48: 577-585.

12. Wolfinger R, O'Connell M. Generalized linear mixed models: a pseudo-likelihood approach. J Statist Comput Simul 1993; 48: 233-243.
13. Asher MI, Pattemore PK, Harrison AC, et al. International comparison of the prevalence of asthma symptoms and bronchial hyperresponsiveness. Am Rev Respir Dis 1988; 138: 524-529.

14. Robertson CF, Bishop J, Sennhauser FH, Mallol J. International comparison of asthma prevalence in children: Australia, Switzerland, Chile. Pediatr Pulmonol 1993; 16: 219-226.

15. Burr ML, Limb ES, Andrae S, Barry DM, Nagel F. Childhood asthma in four countries: a comparative survey. Int $J$ Epidemiol 1994; 23: 341-347.

16. Leung R, Ho P. Asthma, allergy, and atopy in three southeast Asian populations. Thorax 1994; 49: 1205-1210.

17. Bråbäck L, Breborowicz A, Dreborg S, Knutsoson A, Pieklik H, Björkstén B. Atopic sensitisation and respiratory symptoms among Polish and Swedish school children. Clin Exp Allergy 1994; 24: 826-835.

18. Stewart AW, Asher MI, Clayton TO, et al. The effect of season-of-response to ISAAC questions about asthma, rhinitis and eczema in children. Int J Epidemiol 1997; 26: 126-136.

19. Burney PGJ, Luczynska C, Chinn S, Jarvis D. The European Community Respiratory Health Survey. Eur Respir J 1994; 7: 954-960.

20. European Community Respiratory Health Survey (ECRHS). Variations in the prevalence of respiratory symptoms, self-reported asthma attacks, and use of asthma medication in the European Community Respiratory Health Survey (ECRHS). Eur Respir J 1996; 9: 687695.

21. Jenkins MA, Clarke JR, Carlin JB, et al. Validation of questionnaire and bronchial hyperresponsiveness against respiratory physician assessment in the diagnosis of asthma. Int J Epidemiol 1996; 25: 609-616.

22. Ehrlich RI, Toit DD, Jordaan E, Volmink JA, Weinberg EG, Zwarenstein M. Prevalence and reliability of asthma symptoms in primary school children in Cape Town. Int $J$ Epidemiol 1995; 24: 1138-1146.

23. Reidler J, Reade T, Dalton M, Holst D, Robertson C. Hypertonic saline challenge in an epidemiological survey of asthma in children. Am Rev Respir Crit Care Med 1994; 150: 1632-1639.

24. Strachan DP. Epidemiology. In: Silverman M, ed. Childhood Asthma and Other Wheezing Disorders. London, Chapman \& Hall, 1995; pp. 7-31.

25. Bishop J, Robertson CF, Caust J, et al. Concordance between adolescent and parent response to an asthma morbidity questionnaire. Am Rev Respir Dis 1993; 147: A373.

26. Weiss ST, Gold DR. Gender differences in asthma. Pediatr Pulmonol 1995; 19: 153-155.

27. Strachan DP, Limb ES, O'Neill AO, Wells N. A national survey of asthma prevalence, severity and treatment in Great Britain. Arch Dis Child 1994; 70: 174-178.

28. Peat JK, van den Berg RH, Green WF, Mellis CM, Leeder SR, Woolcock AJ. Changing prevalence of asthma in Australian children. BMJ 1994; 308: 15911596.

29. Martinez F. Role of viral infections in the inception of asthma and allergies during childhood: could they be protective? Thorax 1994; 49: 1189-1191.

30. Holt PG. Environmental factors and primary T-cell sensitisation to inhalant allergens in infancy: reappraisal of the role of infections and air pollution. Pediatr Allergy Immunol 1995; 6: 1-10. 